\title{
PRODUCTS OF BLOCK TOEPLITZ OPERATORS
}

\author{
Caixing Gu and Dechao Zheng
}

\begin{abstract}
In this paper we characterize when the product of two block Toeplitz operators is a compact perturbation of a block Toeplitz operator on the Hardy space of the open unit disk. Necessary and sufficient conditions are given for the commutator of two block Toeplitz operators to be compact.
\end{abstract}

\section{Introduction.}

Let $D$ be the open unit disk in the complex plane and $\partial D$ the unit circle. Let $d \sigma(w)$ be the normalized Lebesgue measure on the unit circle. We denote by $L^{2}\left(C^{n}\right)\left(L^{2}\right.$ for $\left.n=1\right)$ the space of $C^{n}$-valued Lebesgue square integrable functions on the unit circle. The Hardy space $H^{2}\left(C^{n}\right)\left(H^{2}\right.$ for $\left.n=1\right)$ is the closed linear span of $C^{n}$-valued analytic polynomials. We observe that $L^{2}\left(C^{n}\right)=L^{2} \otimes C^{n}$ and $H^{2}\left(C^{n}\right)=H^{2} \otimes C^{n}$, where $\otimes$ denotes the Hilbert space tensor product. Let $M_{n \times n}$ be the set of $n \times n$ complex matrices. $L_{n \times n}^{\infty}$ denotes the space of $M_{n \times n}$-valued essentially bounded Lebesgue measurable functions on the unit circle and $H_{n \times n}^{\infty}$ denotes the space of $M_{n \times n}$-valued essentially bounded analytic functions in the disk.

Let $P$ be the projection of $L^{2}\left(C^{n}\right)$ onto $H^{2}\left(C^{n}\right)$. For $F \in L_{n \times n}^{\infty}$, the block Toeplitz operator $T_{F}: H^{2}\left(C^{n}\right) \rightarrow H^{2}\left(C^{n}\right)$ with symbol $F$ is defined by the rule $T_{F} h=P(F h)$. The Hankel operator $H_{F}: H^{2}\left(C^{n}\right) \rightarrow L^{2}\left(C^{n}\right) \ominus H^{2}\left(C^{n}\right)$ with symbol $F$ is defined by $H_{F} h=(I-P)(F h)$. The block Toeplitz operator $T_{F}$ has the following matrix representation:

$$
\left[\begin{array}{cccc}
A_{0} & A_{-1} & A_{-2} & \cdots \\
A_{1} & A_{0} & A_{-1} & \cdots \\
A_{2} & A_{1} & A_{0} & \cdots \\
\vdots & \vdots & \vdots & \vdots
\end{array}\right]
$$

where $A_{i}$ belongs to $M_{n \times n}$. The word "block" refers to the fact that in the above matrix representation the entries are not scalars but linear transformations on $C^{n}$. In this paper the word "block" will often be omitted. For more details on the block Toeplitz operators and Hankel operators, see [7], [9] and [3]. 
If we set $H^{2}\left(C^{n}\right)=H^{2} \oplus \cdots \oplus H^{2}$, then we see that the Toeplitz operator $T_{F}$ has the form

$$
T_{F}=\left[\begin{array}{cccc}
T_{f_{11}} & T_{f_{12}} & \cdots & T_{f_{1 n}} \\
T_{f_{21}} & T_{f_{22}} & \cdots & T_{f_{2 n}} \\
\vdots & \vdots & \vdots & \vdots \\
T_{f_{n 1}} & T_{f_{n 2}} & \cdots & T_{f_{n n}}
\end{array}\right]
$$

and the Hankel operator $H_{F}$ has the form

$$
H_{F}=\left[\begin{array}{cccc}
H_{f_{11}} & H_{f_{12}} & \cdots & H_{f_{1 n}} \\
H_{f_{21}} & H_{f_{22}} & \cdots & H_{f_{2 n}} \\
\vdots & \vdots & \vdots & \vdots \\
H_{f_{n 1}} & H_{f_{n 2}} & \cdots & H_{f_{n n}}
\end{array}\right]
$$

where

$$
F=\left[\begin{array}{cccc}
f_{11} & f_{12} & \cdots & f_{1 n} \\
f_{21} & f_{22} & \cdots & f_{2 n} \\
\vdots & \vdots & \vdots & \vdots \\
f_{n 1} & f_{n 2} & \cdots & f_{n n}
\end{array}\right]
$$

Thus as in the scalar case, the block Toeplitz operators and Hankel operators are connected by the following important relation:

$$
T_{F G}-T_{F} T_{G}=H_{F^{*}}^{*} H_{G} \text {. }
$$

The map $\xi: F \rightarrow T_{F}$, which is called the Toeplitz quantization, carries $L_{n \times n}^{\infty}$ into the $C^{*}$-algebra of bounded operators on $H^{2}\left(C^{n}\right)$. It is a contractive ${ }^{*}$-linear mapping [7]. However it is not multiplicative in general. Indeed Brown and Halmos [4] showed that for scalar functions $f$ and $g, T_{f} T_{g}=T_{f g}$ if and only either $f^{*}$ or $g$ is in $H^{\infty}$. It is not difficult to see that in the matrix case $T_{F} T_{G}=T_{F G}$ still holds if either $F^{*}$ or $G$ is in $H_{n \times n}^{\infty}$. But the converse is not valid in the matrix case. We will characterize $F$ and $G$ such that $T_{F} T_{G}=T_{F G}$; see Theorem 6 below for details. On the other hand, Douglas [7] showed that $\xi$ is actually a cross section for a ${ }^{*}$-homomorphism from the Toeplitz algebra, the $C^{*}$-algebra generated by all bounded Toeplitz operators on $H^{2}\left(C^{n}\right)$, onto $L_{n \times n}^{\infty}$. So modulo the commutator ideal of the Toeplitz algebra, $\xi$ is multiplicative.

The main question to be considered in this paper is when the product of two Toeplitz operators is a compact perturbation of a Toeplitz operator. 
This problem is connected with the spectral theory of Toeplitz operators; see [7], [9] and [3]. It follows from a theorem of Douglas [7] that $T_{F} T_{G}$ can be a compact perturbation of a Toeplitz operator only when it is a compact perturbation of $T_{F G}$. Thus it suffices to study when the semicommutator $T_{F G}-T_{F} T_{G}$ is compact. When $F=f$ and $G=g$ are scalar functions, the problem was solved by Axler, Chang, and Sarason [2] and Volberg [14]. Their beautiful result is that $T_{f g}-T_{f} T_{g}$ is compact if and only if $H^{\infty}[\bar{f}] \cap H^{\infty}[g] \subset H^{\infty}+C(\partial D)$; here $H^{\infty}[g]$ denotes the closed subalgebra of $L^{\infty}$ generated by $H^{\infty}$ and $g$.

Recently, Zheng [15] proved that $T_{f g}-T_{f} T_{g}$ is compact if and only if

$$
\lim _{|z| \rightarrow 1}\left\|H_{\bar{f}} k_{z}\right\|_{2}\left\|H_{g} k_{z}\right\|_{2}=0
$$

here $k_{z}$ denotes the normalized reproducing kernel in $H^{2}$ for point evaluation at $z$. If we write $f=f_{+}+f_{-}$for each $f \in L^{\infty}$ where $f_{+}$and $\overline{f_{-}}$are in $H^{2}$, then the above condition is equivalent to

$$
\lim _{|z| \rightarrow 1}\left|\overline{f_{+}}-\overline{f_{+}}(z)\right|^{2}(z)\left|g_{-}-g_{-}(z)\right|^{2}(z)=0
$$

where $h(z)$ denotes the harmonic extension of $h$ at $z \in D$ for $h \in L^{1}$, via the Poisson integral

$$
h(z)=\int_{\partial D} h(w) \frac{\left(1-|z|^{2}\right)}{|1-w \bar{z}|^{2}} d \sigma(w) .
$$

For the block Toeplitz operators, we will show that $T_{F G}-T_{F} T_{G}$ is compact if and only if

$$
\lim _{|z| \rightarrow 1}\left\|\left[\left|\left(F_{+}\right)^{*}-\left(F_{+}\right)^{*}(z)\right|^{2}(z)\right]^{1 / 2}\left[\left|G_{-}-G_{-}(z)\right|^{2}(z)\right]^{1 / 2}\right\|=0
$$

where we write $F=\left(f_{i j}\right)_{n \times n}$ as $F=F_{+}+F_{-}$with $F_{+}=\left(\left(f_{i j}\right)_{+}\right)_{n \times n}$ and $F_{-}=\left(\left(f_{i j}\right)_{-}\right)_{n \times n}$, and

$$
H(z)=\left(h_{i j}(z)\right)_{n \times n}
$$

if $H=\left(h_{i j}\right)_{n \times n}$. For a matrix $A$, we define $|A|^{2}=A A^{*}$. Several other equivalent conditions, in particular a condition in the spirit of the result of Axler, Chang, and Sarason [2] and Volberg [14], will be given.

In [11] Gorkin and Zheng characterized when the commutator $T_{f} T_{g}-$ $T_{g} T_{f}$ is compact for scalar functions $f$ and $g$. In this paper, by considering block Toeplitz operators, we will give an unified approach for the study of compactness of boths semi-commutators and commutators. Namely, by a theorem of Douglas [7], we have that the commutator $T_{F} T_{G}-T_{G} T_{F}$ of block 
Toeplitz operators $T_{F}$ and $T_{G}$ is compact if and only if $F G=G F$ and the semi-commutator $T_{B C}-T_{B} T_{C}$ is compact, where

$$
B=\left[\begin{array}{cc}
F & -G \\
0 & 0
\end{array}\right], \quad C=\left[\begin{array}{cc}
G & 0 \\
F & 0
\end{array}\right]
$$

see Theorem 7 below for details. Thus we will show the commutator $T_{F} T_{G}-$ $T_{G} T_{F}$ is compact if and only if $F G=G F$ and

$$
\begin{gathered}
\lim _{|z| \rightarrow 1} \|\left[\begin{array}{cc}
\left|\left(F_{+}\right)^{*}-\left(F_{+}\right)^{*}(z)\right|^{2}(z) & -\left(\left(\left(F_{+}\right)^{*}-\left(F_{+}\right)^{*}(z)\right)\right. \\
-\left(\left(\left(G_{+}\right)^{*}-\left(G_{+}\right)^{*}(z)\right)\right. & \left.\cdot\left(G_{+}-G_{+}(z)\right)\right)(z) \\
\left.\cdot\left(F_{+}-F_{+}(z)\right)\right)(z) & \left|\left(G_{+}\right)^{*}-\left(G_{+}\right)^{*}(z)\right|^{2}(z)
\end{array}\right]^{1 / 2} \\
\cdot\left[\begin{array}{cc}
\left|G_{-}-G_{-}(z)\right|^{2}(z) & \left(( G _ { - } - G _ { - } ( z ) ) \left(\left(F_{-}\right)^{*}\right.\right. \\
\left(( F _ { - } - F _ { - } ( z ) ) \left(\left(G_{-}\right)^{*}\right.\right. & \left.\left.-\left(F_{-}\right)^{*}(z)\right)\right)(z) \\
\left.-\left(G_{-}\right)^{*}(z)\right)(z) & \left|F_{-}-F_{-}(z)\right|^{2}(z)
\end{array}\right]^{1 / 2} \|=0 .
\end{gathered}
$$

\section{A necessary condition for compactness.}

In this section we will obtain a necessary condition for the compactness of the semi-commutator $T_{F G}-T_{F} T_{G}$. This will also motivate a necessary and sufficient condition for $T_{F G}-T_{F} T_{G}$ to be zero. The question when $T_{F G}-T_{F} T_{G}$ is zero will be discussed in the next two sections.

First we introduce an antiunitary operator $V$ on $L^{2}$ by defining $(V h)(w)=$ $\overline{w h(w)}$. The operator enjoys many nice properties such as $V^{-1}(I-P) V=P$ and $V=V^{-1}$. These properties leads easily to the relation $V^{-1} H_{f} V=H_{f}^{*}$.

Let $x$ and $y$ be two vectors in $L^{2} . x \otimes y$ is the operator of rank one defined by

$$
(x \otimes y)(f)=\langle f, y\rangle x .
$$

Observe that the norm of the operator $x \otimes y$ is $\|x\|_{2}\|y\|_{2}$.

For $z$ in $D$, let $k_{z}$ be the normalized reproducing kernel $\frac{\left(1-|z|^{2}\right)^{1 / 2}}{(1-\bar{z} w)}$ for point evaluation at $z$, and $\phi_{z}$ the Möbius map on the unit disk,

$$
\phi_{z}(w)=\frac{z-w}{1-\bar{z} w}
$$

$\phi_{z}$ can also be viewed as a function on the unit circle. Let $\Phi_{z}$ denote the function $\operatorname{diag}\left\{\phi_{z}, \cdots, \phi_{z}\right\} \in H_{n \times n}^{\infty}$. The product $T_{\phi_{z}} T_{\overline{\phi_{z}}}$ is the orthogonal 
projection onto $H^{2} \ominus\left\{k_{z}\right\}$. Thus $1-T_{\phi_{z}} T_{\overline{\phi_{z}}}$ is the operator $k_{z} \otimes k_{z}$ of rank one. This leads to the following lemma.

Lemma 1. Let $F=\left(f_{i j}\right)_{n \times n}$ and $G=\left(g_{i j}\right)_{n \times n}$ be in $L_{n \times n}^{\infty}$. Then the operator $H_{F}^{*} H_{G}-T_{\Phi_{z}}^{*} H_{F}^{*} H_{G} T_{\Phi_{z}}$ is anti-unitary equivalent to

$$
\left(\sum_{j=1}^{n} H_{f_{j i}} k_{z} \otimes H_{g_{j k}} k_{z}\right)_{n \times n}
$$

Proof. Let $F=\left(f_{i j}\right)_{n \times n}$ and $G=\left(g_{i j}\right)_{n \times n}$. Then it is easy to check that

$$
H_{F}^{*} H_{G}=\left(\sum_{j=1}^{n} H_{f_{j i}}^{*} H_{g_{j k}}\right)_{n \times n}
$$

and

$$
T_{\Phi_{z}}^{*} H_{F}^{*} H_{G} T_{\Phi_{z}}=\left(\sum_{j=1}^{n} T_{\phi_{z}}^{*} H_{f_{j i}}^{*} H_{g_{j k}} T_{\phi_{z}}\right)_{n \times n} .
$$

So the difference $H_{F}^{*} H_{G}-T_{\Phi_{z}}^{*} H_{F}^{*} H_{G} T_{\Phi_{z}}$ is

$$
\left(\sum_{j=1}^{n}\left[H_{f_{j i}}^{*} H_{g_{j k}}-T_{\phi_{z}}^{*} H_{f_{j i}}^{*} H_{g_{j k}} T_{\phi_{z}}\right]\right)_{n \times n} .
$$

Hence it is sufficient to check that

$$
\sum_{j=1}^{n}\left[H_{f_{j i}}^{*} H_{g_{j k}}-T_{\phi_{z}}^{*} H_{f_{j i}}^{*} H_{g_{j k}} T_{\phi_{z}}\right]
$$

is anti-unitary equivalent to

$$
\sum_{j=1}^{n} H_{f_{j i}} k_{z} \otimes H_{g_{j k}} k_{z}
$$

Applying $V$ and $V^{-1}$ to the difference

$$
\sum_{j=1}^{n}\left[H_{f_{j i}}^{*} H_{g_{j k}}-T_{\phi_{z}}^{*} H_{f_{j i}}^{*} H_{g_{j k}} T_{\phi_{z}}\right]
$$

we have

$$
V^{-1} \sum_{j=1}^{n}\left[H_{f_{j i}}^{*} H_{g_{j k}}-T_{\phi_{z}}^{*} H_{f_{j i}}^{*} H_{g_{j k}} T_{\phi_{z}}\right] V
$$




$$
=V^{-1} \sum_{j=1}^{n}\left[H_{f_{j i}}^{*} H_{g_{j k}}-H_{f_{j i} \phi_{z}}^{*} H_{g_{j k} \phi_{z}}\right] V
$$

Because $V^{-1} H_{f} V=H_{f}^{*}$, the above equality simplifies to

$$
\begin{aligned}
& =V^{-1} \sum_{j=1}^{n}\left[H_{f_{j i}}^{*} H_{g_{j k}}-H_{f_{j i} \phi_{z}}^{*} H_{g_{j k} \phi_{z}}\right] V \\
& =\sum_{j=1}^{n}\left[H_{f_{j i}} H_{g_{j k}}^{*}-H_{f_{j i} \phi_{z}} H_{g_{j k} \phi_{z}}^{*}\right] \\
& =\sum_{j=1}^{n} H_{f_{j i}}\left(1-T_{\phi_{z}} T_{\phi_{z}}^{*}\right) H_{g_{j k}}^{*} .
\end{aligned}
$$

Since $1-T_{\phi_{z}} T_{\phi_{z}}^{*}=k_{z} \otimes k_{z}$, the right hand side of the above equation is

$$
\sum_{j=1}^{n} H_{f_{j i}} k_{z} \otimes H_{g_{j k}} k_{z}
$$

for all $z$ in $D$. This completes the proof of the lemma.

Let trace be the trace on the trace class of operators on $H^{2}$ and $t r$ denote the trace on the $\mathrm{n}$ by $\mathrm{n}$ matrices.

Lemma 2. Let $F$ and $G$ be in $L_{n \times n}^{\infty}$. Let $T=H_{F}^{*} H_{G}-T_{\Phi_{z}}^{*} H_{F}^{*} H_{G} T_{\Phi_{z}}$. Then

$$
\operatorname{trace}\left\{T^{*} T\right\}=\operatorname{tr}\left[\left|F_{-}-F_{-}(z)\right|^{2}(z)\left|G_{-}-G_{-}(z)\right|^{2}(z)\right] .
$$

Proof. Let $F=\left(f_{i j}\right)_{n \times n}$ and $G=\left(g_{i j}\right)_{n \times n}$. By Lemma 1, $H_{F}^{*} H_{G}-$ $T_{\Phi_{z}}^{*} H_{F}^{*} H_{G} T_{\Phi_{z}}$ is anti-unitary equivalent to

$$
T_{1}=\left(\sum_{j=1}^{n} H_{f_{j i}} k_{z} \otimes H_{g_{j k}} k_{z}\right)_{n \times n} .
$$

So we need to computer the trace of the operator $T_{1}^{*} T_{1}$. It is easy to see that

$$
\begin{aligned}
T_{1}^{*} T_{1} & =\left(\sum_{j_{1}}\left(\sum_{j} H_{f_{j j_{1}}} k_{z} \otimes H_{g_{j k}} k_{z}\right)^{*}\left(\sum_{\mu} H_{f_{\mu j_{1}}} k_{z} \otimes H_{g_{\mu l}} k_{z}\right)\right) \\
& =\left(\sum_{j_{1}} \sum_{j} \sum_{\mu}\left\langle H_{f_{\mu j_{1}}} k_{z}, H_{f_{j j_{1}}} k_{z}\right\rangle H_{g_{j k}} k_{z} \otimes H_{g_{\mu l}} k_{z}\right),
\end{aligned}
$$


where the second equality above follows from the fact that

$$
\left(x_{1} \otimes y_{1}\right)^{*}\left(x_{2} \otimes y_{2}\right)=\left\langle x_{2}, x_{1}\right\rangle y_{1} \otimes y_{2} .
$$

Thus

$$
\operatorname{trace}\left\{T_{1}^{*} T_{1}\right\}=\sum_{l} \sum_{j_{1}} \sum_{j} \sum_{\mu}\left\langle H_{f_{\mu_{1}}} k_{z}, H_{f_{j j_{1}}} k_{z}\right\rangle\left\langle H_{g_{j l}} k_{z}, H_{g_{\mu l}} k_{z}\right\rangle .
$$

If we write $f_{i j}=\left(f_{i j}\right)_{+}+\left(f_{i j}\right)_{-}$and $g_{i j}=\left(g_{i j}\right)_{+}+\left(g_{i j}\right)_{-}$for $\left(f_{i j}\right)_{+},\left(g_{i j}\right)_{+} \in$ $H^{2}$ and $\left(f_{i j}\right)_{-},\left(g_{i j}\right)_{-} \in \overline{z H^{2}}$, then $H_{f_{i j}} k_{z}=\left(\left(f_{i j}\right)_{-}-\left(f_{i j}\right)_{-}(z)\right) k_{z}$ and $H_{g_{i j}} k_{z}=\left(\left(g_{i j}\right)_{-}-\left(g_{i j}\right)_{-}(z)\right) k_{z}$. Therefore, by a change of the order of summations, we have

$$
\begin{aligned}
& \operatorname{trace}\left\{T_{1}^{*} T_{1}\right\} \\
& \begin{aligned}
= & \sum_{l} \sum_{j_{1}} \sum_{j} \sum_{\mu}\left\langle\left[\left(f_{\mu j_{1}}\right)_{-}-\left(f_{\mu j_{1}}\right)_{-}(z)\right] k_{z},\left[\left(f_{j j_{1}}\right)_{-}-\left(f_{j j_{1}}\right)_{-}(z)\right] k_{z}\right\rangle \\
& \cdot\left\langle\left[\left(g_{j l}\right)_{-}-\left(g_{j l}\right)_{-}(z)\right] k_{z},\left[\left(g_{\mu l}\right)_{-}-\left(g_{\mu l}\right)_{-}(z)\right] k_{z}\right\rangle \\
= & \sum_{\mu} \sum_{j}\left(\sum_{j_{1}}\left\langle\left[\left(f_{\mu j_{1}}\right)_{-}-\left(f_{\mu j_{1}}\right)_{-}(z)\right] k_{z},\left[\left(f_{j j_{1}}\right)_{-}-\left(f_{j j_{1}}\right)_{-}(z)\right] k_{z}\right\rangle\right) \\
& \cdot\left(\sum_{l}\left\langle\left[\left(g_{j l}\right)_{-}-\left(g_{j l}\right)_{-}(z)\right] k_{z},\left[\left(g_{\mu l}\right)_{-}-\left(g_{\mu l}\right)_{-}(z)\right] k_{z}\right\rangle\right) .
\end{aligned}
\end{aligned}
$$

Note that

$$
\begin{aligned}
& \left(\sum_{j_{1}}\left\langle\left[\left(f_{\mu j_{1}}\right)_{-}-\left(f_{\mu j_{1}}\right)_{-}(z)\right] k_{z},\left[\left(f_{j j_{1}}\right)_{-}-\left(f_{j j_{1}}\right)_{-}(z)\right] k_{z}\right\rangle\right)_{n \times n} \\
& =\left|F_{-}-F_{-}(z)\right|^{2}(z),
\end{aligned}
$$

and similarly

$$
\begin{aligned}
& \left(\sum_{l}\left\langle\left[\left(g_{j l}\right)_{-}-\left(g_{j l}\right)_{-}(z)\right] k_{z},\left[\left(g_{\mu l}\right)_{-}-\left(g_{\mu l}\right)_{-}(z)\right] k_{z}\right\rangle\right)_{n \times n} \\
& =\left|G_{-}-G_{-}(z)\right|^{2}(z) .
\end{aligned}
$$

Hence

$$
\begin{aligned}
\operatorname{trace}\left\{T_{1}^{*} T_{1}\right\} & =\sum_{u} \sum_{j}\left(\left|F_{-}-F_{-}(z)\right|^{2}(z)\right)_{\mu j}\left(\left|G_{-}-G_{-}(z)\right|^{2}(z)\right)_{j \mu} \\
& =\sum_{u}\left[\left|F_{-}-F_{-}(z)\right|^{2}(z)\left(\left|G_{-}-G_{-}(z)\right|^{2}(z)\right)\right]_{\mu \mu}
\end{aligned}
$$




$$
=\operatorname{tr}\left[\left|F_{-}-F_{-}(z)\right|^{2}(z)\left|G_{-}-G_{-}(z)\right|^{2}(z)\right]
$$

Here we note that for a matrix $A,|A|^{2}=A A^{*}$ and $(A)_{i j}$ denotes the $(i, j)-t h$ entry of $A$. This completes the proof of this lemma.

Theorem 3. Let $F$ and $G$ be in $L_{n \times n}^{\infty}$. If $H_{F}^{*} H_{G}$ is compact, then

$$
\lim _{|z| \rightarrow 1}\left\|\left\{\left[\left|F_{-}-F_{-}(z)\right|^{2}(z)\right]^{1 / 2}\left[\left|G_{-}-G_{-}(z)\right|^{2}(z)\right]^{1 / 2}\right\}\right\|=0 .
$$

Proof. Set $H^{2}\left(C^{n}\right)=H^{2} \oplus \cdots \oplus H^{2}$. Let $\left(A_{i j}\right):=H_{F}^{*} H_{G}$ be the operatorvalued $n \times n$ matrix representation of $H_{F}^{*} H_{G}$ with respect to the above decomposition of $H^{2}\left(C^{n}\right)$. Since $H_{F}^{*} H_{G}$ compact, each entry $A_{i j}$ of $H_{F}^{*} H_{G}$ is compact on $H^{2}$, by Lemma 2 [15], we have

$$
\lim _{|z| \rightarrow 1}\left\|A_{i j}-T_{\phi_{z}}^{*} A_{i j} T_{\phi_{z}}\right\|=0
$$

Hence

$$
\lim _{|z| \rightarrow 1}\left\|H_{F}^{*} H_{G}-T_{\Phi_{z}}^{*} H_{F}^{*} H_{G} T_{\Phi_{z}}\right\|=0 .
$$

By Lemma $1, H_{F}^{*} H_{G}-T_{\Phi_{z}}^{*} H_{F}^{*} H_{G} T_{\Phi_{z}}$ is a finite rank operator. Therefore, we have

$$
\lim _{|z| \rightarrow 1} \operatorname{trace}\left\{\left(H_{F}^{*} H_{G}-T_{\Phi_{z}}^{*} H_{F}^{*} H_{G} T_{\Phi_{z}}\right)^{*}\left(H_{F}^{*} H_{G}-T_{\Phi_{z}}^{*} H_{F}^{*} H_{G} T_{\Phi_{z}}\right)\right\}=0
$$

because the norm of a finite rank positive operator is equivalent to its trace. By Lemma 2, we obtain

$$
\lim _{|z| \rightarrow 1} \operatorname{tr}\left[\left|F_{-}-F_{-}(z)\right|^{2}(z)\left|G_{-}-G_{-}(z)\right|^{2}(z)\right]=0 .
$$

On the other hand,

$$
\begin{aligned}
& \operatorname{tr}\left[\left|F_{-}-F_{-}(z)\right|^{2}(z)\left|G_{-}-G_{-}(z)\right|^{2}(z)\right] \\
& =\operatorname{tr}\left\{\left[\left|F_{-}-F_{-}(z)\right|^{2}(z)\right]^{1 / 2}\left[\left|G_{-}-G_{-}(z)\right|^{2}(z)\right]\left[\left|F_{-}-F_{-}(z)\right|^{2}(z)\right]^{1 / 2}\right\} \\
& =\operatorname{tr}\left\{\left[\left|F_{-}-F_{-}(z)\right|^{2}(z)\right]^{1 / 2}\left[\left|G_{-}-G_{-}(z)\right|^{2}(z)\right]^{1 / 2}\right. \\
& \left.\quad \cdot\left\{\left[\left|F_{-}-F_{-}(z)\right|^{2}(z)\right]^{1 / 2}\left[\left|G_{-}-G_{-}(z)\right|^{2}(z)\right]^{1 / 2}\right\}^{*}\right\} .
\end{aligned}
$$

As is well-known, for all $n \times n$ matrices $A$,

$$
\operatorname{tr} A^{*} A \geq C\|A\|^{2}
$$

for some constant $C>0$. Hence we conclude

$$
\lim _{|z| \rightarrow 1}\left\|\left\{\left[\left|F_{-}-F_{-}(z)\right|^{2}(z)\right]^{1 / 2}\left[\left|G_{-}-G_{-}(z)\right|^{2}(z)\right]^{1 / 2}\right\}\right\|=0 .
$$

This completes the proof of the theorem. 


\section{Finite sum of the product of Hankel operators.}

In this section we will discuss when the finite sum of the products of Hankel operators with scalar symbols is zero. This is needed in the next section for characterizing $F$ and $G$ in $L_{n \times n}^{\infty}$ such that $T_{F G}=T_{F} T_{G}$.

Let $M_{n \times n}$ be the set of $n \times n$ matrices. Let $A=\left[a_{i j}\right] \in M_{n \times n}$, define

$$
\|A\|_{\infty}=\sup _{1 \leq i, j \leq n}\left|a_{i j}\right|
$$

and $\left(M_{n \times n}\right)_{1}$ denotes the closed unit ball of $M_{n \times n}$ in the above norm. Let $P_{n}$ be the set of $n \times n$ permutation matrices.

Proposition 4 Let $f_{k}=\left(f_{k 1}, \ldots, f_{k n}\right)^{T}$ for $k=1, \ldots, m$ and $g=\left(g_{1}, \ldots\right.$, $\left.g_{n}\right)^{T} \in L^{2}\left(C^{n}\right)$. Let

$$
S_{k n}:=f_{k 1} \otimes g_{1}+\cdots+f_{k n} \otimes g_{n}, \quad k=1, \ldots, m .
$$

Then $S_{k n}=0$ for all $k=1, \ldots, m$ if and only if there are a matrix $A \in$ $\left(M_{n \times n}\right)_{1}$ and a permutation matrix $R$ such that

$$
(R-A) f_{k}=0, k=1, \ldots, m \quad \text { and } \quad A^{*} g=0 .
$$

Proof. We first prove that $S_{k n}=0, k=1, \ldots, m$ if and only if there are a matrix $A_{0} \in\left(M_{n \times n}\right)_{1}$ and a permutation $\sigma$ such that

$$
\left(I-A_{0}\right) f_{k \sigma}=0, k=1, \ldots, m \quad \text { and } \quad A_{0}^{*} g_{\sigma}=0,
$$

where $f_{k \sigma}=\left(f_{k \sigma(1)}, \ldots, f_{k \sigma(n)}\right)^{T}$. For any $A \in\left(M_{n \times n}\right)_{1}$, any permutation $\sigma$, set

$$
\begin{aligned}
x_{k}:=\left(x_{k 1}, \cdots, x_{k n}\right)^{T}=(I-A) f_{k \sigma}, \quad k & =1, \ldots, m \\
& \text { and } y:=\left(y_{1}, \ldots, y_{n}\right)^{T}=A^{*} g_{\sigma},
\end{aligned}
$$

then we have

$S_{k n}=\sum f_{k \sigma_{i}} \otimes g_{\sigma_{i}}=x_{k 1} \otimes g_{\sigma(1)}+\cdots+x_{k n} \otimes g_{\sigma(n)}+f_{k \sigma(1)} \otimes y_{1}+\cdots+f_{k \sigma(n)} \otimes y_{n}$.

The sufficiency follows from the above relation. To prove the necessity we use induction. It is clear that for $n=1$, the result is true with $A=1$ or $A=0$. Now assume the result is true for $n-1$. Without loss of generality, assume that

$$
\max _{1 \leq i \leq n}\left\|g_{i}\right\|_{2}=\left\|g_{j}\right\|_{2}>0
$$


for some $j$. Note that if $S_{k n}=0, k=1, \ldots, m$, then

$$
S_{k n} g_{j}=\sum_{i=1}^{n}\left\langle g_{j}, g_{i}\right\rangle f_{i}=0, \quad k=1, \ldots, m
$$

That is

$$
f_{k j}+\sum_{i \neq j} a_{j} f_{k i}=0
$$

where $a_{i}=\left\langle g_{i}, g_{j}\right\rangle /\left\langle g_{i}, g_{j}\right\rangle,\left|a_{i}\right| \leq 1$ for $i \neq j$. Now we rewrite $S_{k n}$ as

$$
S_{k n}=\left(f_{k j}+\sum_{i \neq j} a_{j} f_{k i}\right) \otimes g_{j}+\sum_{i \neq j} f_{k i} \otimes\left(g_{i}-\overline{a_{j}} g_{j}\right) .
$$

From above analysis we have that

$$
\sum_{i \neq j} f_{k i} \otimes\left(g_{i}-\overline{a_{j}} g_{j}\right)=0, k=1, \ldots, m
$$

By induction there exist $A_{1} \in\left(M_{n-1 \times n-1}\right)_{1}$ and a permutation $\omega$ of $\{1, \ldots$, $j-1, j+1, \ldots, n\}$ such that

$$
\begin{aligned}
&\left(I-A_{1}\right)\left(f_{k \omega(1)}, \ldots,\right.\left.f_{k \omega(j-1)}, f_{k \omega(j+1)}, \ldots, f_{k \omega(n)}\right)^{T}=0, \quad k=1, \ldots, m, \\
& A_{1}^{*}\left(g_{\omega(1)}-\overline{a_{\omega(1)}} g_{\omega(j)}, \ldots, g_{\omega(j-1)}-\overline{a_{\omega(j-1)}} g_{\omega(j)},\right. \\
&\left.g_{\omega(j+1)}-\overline{a_{\omega(j+1)}} g_{\omega(j)}, \ldots, g_{\omega(n)}-\overline{a_{\omega(n)}} g_{\omega(j)}\right)^{T}=0 .
\end{aligned}
$$

Let

$$
A_{0}=\left[\begin{array}{cc}
0 & a \\
0 & A_{1}
\end{array}\right], \quad \text { where } \quad a=\left[-a_{\omega(1)}, \ldots,-a_{\omega(j-1)},-a_{\omega(j+1)}, \ldots,-a_{\omega(n)}\right] .
$$

Take $\sigma$ to be such that $\sigma(1)=j, \sigma(i)=\omega(i+1)$ for $2 \leq i \leq j-1$ and $\sigma(i)=\omega(i)$ for $j+1 \leq i \leq n$. It is easy to check that such $A_{0}$ and $\sigma$ are what we need. Now let $R$ be the permuation matrix such that $f_{k \sigma}=R f_{k}$ for all $k=1, \ldots, m$ and $A=R^{*} A_{0}$, then it is easy to check that for such $A$ and $R$

$$
(R-A) f_{k}=0, k=1, \ldots, m \quad \text { and } \quad A^{*} g=0
$$

if and only if

$$
\left(I-A_{0}\right) f_{k \sigma}=0, k=1, \ldots, m \quad \text { and } \quad A_{0}^{*} g_{\sigma}=0 .
$$

The proof is complete. 
Next we discuss when the finite sum of the products of Hankel operators is zero.

Theorem 5. Let $f_{k}=\left(f_{k 1}, \ldots, f_{k n}\right)^{T}, k=1, \ldots, m$ and $g=\left(g_{1}, \ldots, g_{n}\right)^{T}$ for $f_{i j}$ and $g_{i}$ in $L^{\infty}$. Let

$$
T_{k n}:=\sum_{i=1}^{n} H_{f_{k i}}^{*} H_{g_{i}}, \quad k=1, \ldots, m .
$$

Then $T_{k n}=0$ for all $k=1, \ldots, m$ if and only if there are a matrix $A \in$ $\left(M_{n \times n}\right)_{1}$ and a permutation matrix $R$ such that

$$
(R-A) f_{k} \in H_{n \times 1}^{\infty}, k=1, \ldots, m \quad \text { and } \quad A^{*} g \in H_{n \times 1}^{\infty} .
$$

Proof. To prove the necessity, we recall the following identity proved in Lemma 1.

$$
\begin{gathered}
V^{-1}\left[\sum_{i=1}^{n}\left(H_{f_{k i}}^{*} H_{g_{i}}-T_{\phi_{z}}^{*} H_{f_{k i}} H_{g_{i}} T_{\phi_{z}}\right)\right] V \\
=\sum_{i=1}^{n} H_{f_{k i}} k_{z} \otimes H_{g_{i}} k_{z}, \quad k=1, \ldots, m .
\end{gathered}
$$

Therefore if $\sum_{i=1}^{n} H_{f_{k i}}^{*} H_{g_{i}}=0$, then

$$
\sum_{i=1}^{n} H_{f_{k i}} k_{z} \otimes H_{g_{i}} k_{z}=0
$$

In particular for $z=0$ (i.e., $k_{0}=1$ ), by Proposition 4 , the above equation implies that there exist a matrix $A \in\left(M_{n \times n}\right)_{1}$ and a permutation matrix $R$ such that

$$
\begin{aligned}
&(R-A)\left[H_{f_{k 1}} 1, \ldots, H_{f_{k n}} 1\right]^{T}=0, \quad k=1, \ldots, m \\
& \quad \text { and } A^{*}\left[H_{g_{1}} 1, \ldots, H_{g_{n}} 1\right]^{T}=0 .
\end{aligned}
$$

That is (1) holds. To prove the sufficiency, as in the proof of Proposition 4, we note that for any $A \in\left(M_{n \times n}\right)_{1}$, any permutation matrix $R$, if we set

$$
x_{k}=\left(x_{k 1}, \ldots, x_{k n}\right)^{T}=(R-A) f_{k \sigma} \text { and } y=\left(y_{1}, \ldots, y_{n}\right)^{T}=A^{*} g_{\sigma},
$$

then we have

$$
\begin{aligned}
T_{k n}=H_{x_{k 1}}^{*} H_{g_{1}}+\cdots+H_{x_{k n}}^{*} H_{g_{n}}+H_{f_{k 1}}^{*} H_{y_{1}}+\cdots+H_{f_{k n}}^{*} H_{y_{n}}, & k=1, \ldots, m .
\end{aligned}
$$

The above formula and the fact that $H_{b}$ is zero when $b \in H^{\infty}$ prove the sufficiency part of our theorem. 


\section{Zero semi-commutator or commutator.}

Brown and Halmos [4] showed that the semi-commutator $T_{\phi \psi}-T_{\phi} T_{\psi}$ is zero exactly when either $\phi$ or $\psi$ is analytic for the scalar functions $\phi$ and $\psi$. Halmos [12] also characterized when the commutator $T_{\phi} T_{\psi}-T_{\psi} T_{\phi}$ is zero. In this section, we will characterize when the semi-commutator $T_{F G}-T_{F} T_{G}$ or the commutator $T_{F} T_{G}-T_{G} T_{F}$ is zero for block Toeplitz operators with matrix symbols $F$ and $G$.

Let $E_{j}$ be the $n \times n$ matrix unit with $(j, j)$-th entry equal to one and all other entries equal to zero. Note that for a $m \times n$ matrix $B, B E_{j}$ is basically the j-th column of $B$.

Theorem 6. Let $F, G \in L_{n \times n}^{\infty}$. The following are equivalent.

(1) The semi-commutator $T_{F} T_{G}-T_{F G}\left(=-H_{F^{*}}^{*} H_{G}\right)$ is zero.

(2) There exist matrices $A_{j} \in\left(M_{n \times n}\right)_{1}$ and $R_{j} \in P_{n}, j=1, \ldots, n$ such that

$$
\left(R_{j}-A_{j}\right) F^{*} \in H_{n \times n}^{\infty} \quad \text { and } \quad A_{j}^{*} G E_{j} \in H_{n \times n}^{\infty}, j=1, \ldots, n .
$$

$$
\left[\left|\left(F_{+}\right)^{*}-\left(F_{+}\right)^{*}(z)\right|^{2}(z)\right]^{1 / 2}\left[\left|G_{-}-G_{-}(z)\right|^{2}(z)\right]^{1 / 2}=0
$$

for all $z \in D$.

$$
\left[\left|\left(F_{+}\right)^{*}-\left(F_{+}\right)^{*}(z)\right|^{2}(z)\right]^{1 / 2}\left[\left|G_{-}-G_{-}(z)\right|^{2}(z)\right]^{1 / 2}=0
$$

for some $z \in D$.

Proof. (1) $\Longleftrightarrow(2)$. Let $F=\left(f_{i j}\right)$ and $G=\left(g_{i j}\right)$. Since $T_{F} T_{G}-T_{F G}=H_{F^{*}}^{*} H_{G}$, $T_{F} T_{G}-T_{F G}=0$ if and only if for each $j=1, \ldots, n$

$$
\sum_{i=1}^{n} H_{\bar{f}_{i k}}^{*} H_{g_{i j}}=0, \quad k=1, \ldots, n
$$

By Theorem 5 , this is equivalent to that for each $j=1, \ldots, n$, there exist matrix $A_{j} \in\left(M_{n \times n}\right)_{1}$ and $R_{j} \in P_{n}$ such that

$$
\left(R_{j}-A_{j}\right) F^{*} \in H_{n \times n}^{\infty} \quad \text { and } \quad A_{j}^{*} G E_{j} \in H_{n \times n}^{\infty} .
$$

$(1) \Longrightarrow(3)$. Since $T_{F} T_{G}-T_{F G}=H_{F^{*}}^{*} H_{G}$, by Lemma 2 , for all $z \in D$, we have

$$
\begin{aligned}
& \operatorname{trace}\left\{\left[H_{F^{*}}^{*} H_{G}-T_{\Phi_{z}}^{*}\left[H_{F^{*}}^{*} H_{G}\right] T_{\Phi_{z}}\right]^{*}\left[H_{F^{*}}^{*} H_{G}-T_{\Phi_{z}}^{*}\left[H_{F^{*}}^{*} H_{G}\right] T_{\Phi_{z}}\right]\right\} \\
& =\operatorname{tr}\left[\left(\left|\left(F_{+}\right)^{*}-\left(F_{+}\right)^{*}(z)\right|^{2}(z)\right)\left(\left|G_{-}-G_{-}(z)\right|^{2}(z)\right)\right]
\end{aligned}
$$




$$
\begin{aligned}
= & \operatorname{tr}\left[\left[\left|\left(F_{+}\right)^{*}-\left(F_{+}\right)^{*}(z)\right|^{2}(z)\right]^{1 / 2}\left[\left|G_{-}-G_{-}(z)\right|^{2}(z)\right]^{1 / 2}\right. \\
& \left.\left.\cdot\left[\left|\left(F_{+}\right)^{*}-\left(F_{+}\right)^{*}(z)\right|^{2}(z)\right]^{1 / 2}\left[\left|G_{-}-G_{-}(z)\right|^{2}(z)\right]^{1 / 2}\right)^{*}\right] .
\end{aligned}
$$

Hence

$$
\left[\left|\left(F_{+}\right)^{*}-\left(F_{+}\right)^{*}(z)\right|^{2}(z)\right]^{1 / 2}\left[\left|G_{-}-G_{-}(z)\right|^{2}(z)\right]^{1 / 2}=0 .
$$

$(3) \Longrightarrow(4)$. This is obvious.

$(4) \Longrightarrow(1)$. For a given $z \in D$, define a unitary operator $U_{z}$ on $H^{2}$ by $U_{z} h=h \circ \phi_{z} k_{z}$. Let $\mathcal{U}_{z}=\operatorname{diag}\left\{U_{z}, \ldots, U_{z}\right\}$. Then it is easy to check that

$$
\mathcal{U}_{z}^{*} T_{F} \mathcal{U}_{z}=T_{F \circ \phi_{z}} \text {. }
$$

Therefore

$$
\begin{aligned}
& \mathcal{U}_{z}^{*}\left[H_{F^{*}}^{*} H_{G}-T_{\Phi_{z}}^{*} H_{F^{*}}^{*} H_{G} T_{\Phi_{z}}\right] \mathcal{U}_{z} \\
& =\left[H_{F^{*} \circ \phi_{z}}^{*} H_{G \circ \phi_{z}}-T_{\Phi_{0}}^{*} H_{F^{*} \circ \phi_{z}}^{*} H_{G \circ \phi_{z}} T_{\Phi_{0}}\right] .
\end{aligned}
$$

So it is sufficient to prove that $H_{F^{*}}^{*} H_{G}=0$ if we assume that

$$
\left[\left|\left(F_{+}\right)^{*}-\left(F_{+}\right)^{*}(0)\right|^{2}(0)\right]^{1 / 2}\left[\left|G_{-}-G_{-}(0)\right|^{2}(0)\right]^{1 / 2}=0 .
$$

By Lemma 2, we have

$$
\begin{aligned}
& \operatorname{trace}\left\{\left[H_{F^{*}}^{*} H_{G}-T_{\Phi_{0}}^{*} H_{F^{*}}^{*} H_{G} T_{\Phi_{0}}\right]^{*}\left[H_{F^{*}}^{*} H_{G}-T_{\Phi_{0}}^{*} H_{F^{*}}^{*} H_{G} T_{\Phi_{0}}\right]\right\} \\
& =\operatorname{tr}\left[\left[\left|\left(F_{+}\right)^{*}-\left(F_{+}\right)^{*}(0)\right|^{2}(0)\right]^{1 / 2}\left[\left|G_{-}-G_{-}(0)\right|^{2}(0)\right]^{1 / 2}\right. \\
& \left.\cdot\left(\left[\left|\left(F_{+}\right)^{*}-\left(F_{+}\right)^{*}(0)\right|^{2}(0)\right]^{1 / 2}\left[\left|G_{-}-G_{-}(0)\right|^{2}(0)\right]^{1 / 2}\right)^{*}\right] .
\end{aligned}
$$

So

$$
H_{F^{*}}^{*} H_{G}-T_{\Phi_{0}}^{*} H_{F^{*}}^{*} H_{G} T_{\Phi_{0}}=0 .
$$

Thus it follows from a theorem [6] that there is a matrix valued function $M$ in $L_{n \times n}^{\infty}$ such that $H_{F^{*}}^{*} H_{G}=T_{M}$. But by the Douglas theorem [7] we have that $M=0$. Hence $H_{F^{*}}^{*} H_{G}=0$.

Next we study the commutator $T_{F} T_{G}-T_{G} T_{F}$ by reducing it to the semicommutator case. To see this, first note that

$$
\begin{aligned}
T_{F} T_{G}-T_{G} T_{F} & =T_{F} T_{G}-T_{F G}+T_{G F}-T_{G} T_{F}+T_{(F G-G F)} \\
& =-\left(H_{F^{*}}^{*} H_{G}-H_{G^{*}}^{*} H_{F}\right)+T_{(F G-G F)} .
\end{aligned}
$$

Let

$$
B=\left[\begin{array}{cc}
F & -G \\
0 & 0
\end{array}\right], \quad C=\left[\begin{array}{cc}
G & 0 \\
F & 0
\end{array}\right]
$$


A simple calculation gives that

$$
H_{B^{*}}^{*} H_{C}=\left[\begin{array}{cc}
H_{F^{*}}^{*} H_{G}-H_{G^{*}}^{*} H_{F} & 0 \\
0 & 0
\end{array}\right] .
$$

Therefore, by the Douglas theorem [7], $T_{F} T_{G}-T_{G} T_{F}=0$ if and only if $H_{B^{*}}^{*} H_{C}=0$ and $F G=G F$. A straightforward computation shows that

$$
\begin{aligned}
& \left|\left(B_{+}\right)^{*}-\left(B_{+}\right)^{*}(z)\right|^{2}(z) \\
& =\left[\begin{array}{cc}
\left|\left(F_{+}\right)^{*}-\left(F_{+}\right)^{*}(z)\right|^{2}(z) & -\left(\left(\left(F_{+}\right)^{*}-\left(F_{+}\right)^{*}(z)\right)\right. \\
-\left(\left(\left(G_{+}\right)^{*}-\left(G_{+}\right)^{*}(z)\right)\right. & \left.\cdot\left(G_{+}-G_{+}(z)\right)\right)(z) \\
\left.\cdot\left(F_{+}-F_{+}(z)\right)\right)(z) & \left|\left(G_{+}\right)^{*}-\left(G_{+}\right)^{*}(z)\right|^{2}(z)
\end{array}\right] . \\
& \left|C_{-}-C_{-}(z)\right|^{2}(z) \\
& =\left[\begin{array}{cc}
\left|G_{-}-G_{-}(z)\right|^{2}(z) & \left(( G _ { - } - G _ { - } ( z ) ) \left(\left(F_{-}\right)^{*}\right.\right. \\
\left(( F _ { - } - F _ { - } ( z ) ) \left(\left(G_{-}\right)^{*}\right.\right. & \left.\left.-\left(F_{-}\right)^{*}(z)\right)\right)(z) \\
\left.\left.-\left(G_{-}\right)^{*}(z)\right)\right)(z) & \left|F_{-}-F_{-}(z)\right|^{2}(z)
\end{array}\right] .
\end{aligned}
$$

This leads to the following result.

Theorem 7. Let $F, G \in L_{n \times n}^{\infty}$. The following are equivalent.

(1) The commutator $T_{F} T_{G}-T_{G} T_{F}$ is zero.

(2) $G F=F G$ and there exist matrices $A_{j} \in\left(M_{2 n \times 2 n}\right)_{1}$ and $R_{j} \in P_{2 n}, j=$ $1, \ldots, n$ such that

$$
\left(R_{j}-A_{j}\right)\left[\begin{array}{c}
F^{*} \\
-G^{*}
\end{array}\right] \in H_{2 n \times n}^{\infty} \text { and } A_{j}^{*}\left[\begin{array}{c}
G \\
F
\end{array}\right] E_{j} \in H_{2 n \times n}^{\infty}, j=1, \ldots, n .
$$

(3) $\quad G F=F G$. And

$$
\begin{aligned}
& {\left[\begin{array}{cc}
\left|\left(F_{+}\right)^{*}-\left(F_{+}\right)^{*}(z)\right|^{2}(z) & -\left(\left(\left(F_{+}\right)^{*}-\left(F_{+}\right)^{*}(z)\right)\right. \\
-\left(\left(\left(G_{+}\right)^{*}-\left(G_{+}\right)^{*}(z)\right)\right. & \left.\cdot\left(G_{+}-G_{+}(z)\right)\right)(z) \\
\left.\cdot\left(F_{+}-F_{+}(z)\right)\right)(z) & \left|\left(G_{+}\right)^{*}-\left(G_{+}\right)^{*}(z)\right|^{2}(z)
\end{array}\right]^{1 / 2}} \\
& \cdot\left[\begin{array}{cc}
\left|G_{-}-G_{-}(z)\right|^{2}(z) & \left(( G _ { - } - G _ { - } ( z ) ) \left(\left(F_{-}\right)^{*}\right.\right. \\
\left(( F _ { - } - F _ { - } ( z ) ) \left(\left(G_{-}\right)^{*}\right.\right. & \left.\left.-\left(F_{-}\right)^{*}(z)\right)\right)(z) \\
\left.\left.-\left(G_{-}\right)^{*}(z)\right)\right)(z) & \left|F_{-}-F_{-}(z)\right|^{2}(z)
\end{array}\right]^{1 / 2} \\
& =0
\end{aligned}
$$

for some $z \in D$. 
We remark that as in Theorem 6, the matrix in the statement (3) of Theorem 7 is zero for some $z \in D$ if and only if it is zero for all $z \in D$.

An operator $A$ is said to be normal if $A A^{*}-A^{*} A=0$. We observe that by taking $G=F^{*}$ in Theorem 7 and noting that $T_{F}^{*}=T_{F^{*}}$, we have the following characterization of normal block Toeplitz operators.

Corollary 8. Let $F \in L_{n \times n}^{\infty}$. The following are equivalent.

(1) $T_{F}$ is normal.

(2) $\quad F^{*} F=F F^{*}$. And there exist matrices $A_{j} \in\left(M_{2 n \times 2 n}\right)_{1}$ and $R_{j} \in$ $P_{2 n}, j=1, \ldots, n$ such that

$$
\left(R_{j}-A_{j}\right)\left[\begin{array}{c}
F^{*} \\
-F
\end{array}\right] \in H_{2 n \times n}^{\infty} \text { and } A_{j}^{*}\left[\begin{array}{c}
F^{*} \\
F
\end{array}\right] E_{j} \in H_{2 n \times n}^{\infty}, \quad j=1, \ldots, n .
$$

$$
\begin{aligned}
F^{*} F= & F F^{*} \text {. And } \\
& {\left[\begin{array}{ll}
\left|\left(F_{+}\right)^{*}-\left(F_{+}\right)^{*}(z)\right|^{2}(z) & -\left(\left(F_{-}-F_{-}(z)\right)\right. \\
-\left(\left(\left(F_{+}\right)^{*}-\left(F_{+}\right)^{*}(z)\right)\right. & \left.\cdot\left(F_{+}-F_{+}(z)\right)\right)(z) \\
\left.\cdot\left(\left(F_{-}\right)^{*}-\left(F_{-}\right)^{*}(z)\right)\right)(z) & \left|F_{-}-F_{-}(z)\right|^{2}(z)
\end{array}\right]^{1 / 2} } \\
& \cdot\left[\begin{array}{ll}
\left|\left(F_{+}\right)^{*}-\left(F_{+}\right)^{*}(z)\right|^{2}(z) & \left(\left(F_{-}-F_{-}(z)\right)\right. \\
\left(\left(\left(F_{+}\right)^{*}-\left(F_{+}\right)^{*}(z)\right)\right. & \left.\cdot\left(F_{+}(z)\right)\right)(z) \\
\left.\cdot\left(\left(F_{-}\right)^{*}-\left(F_{-}\right)^{*}(z)\right)\right)(z) & \left|F_{-}-F_{-}(z)\right|^{2}(z)
\end{array}\right]^{1 / 2} \\
= & 0
\end{aligned}
$$

for some $z \in D$.

Proof. Let $\left|\left(B_{+}\right)^{*}-\left(B_{+}\right)^{*}(z)\right|^{2}(z)$ and $\left|C_{-}-C_{-}(z)\right|^{2}$ be defined by (4) and (5) with $G=F^{*}$. The corollary follows from Theorem 7 by noting that

$$
\left(G_{+}\right)^{*}-\left(G_{+}\right)^{*}(z)=F_{-}-F_{-}(z), \quad G_{-}-G_{-}(z)=\left(F_{+}\right)^{*}-\left(F_{+}\right)^{*}(z) .
$$

\section{A distribution function inequality.}

Recall that a necessary condition for the compactness of the semi-commutator $T_{F G}-T_{F} T_{G}$ is obtained in Theorem 3. Namely, the compactness of $T_{F G}-$ $T_{F} T_{G}$ implies that

$$
\lim _{|z| \rightarrow 1}\left\|\left[\left|\left(F_{+}\right)^{*}-\left(F_{+}\right)^{*}(z)\right|^{2}(z)\right]^{1 / 2}\left[\left|G_{-}-G_{-}(z)\right|^{2}(z)\right]^{1 / 2}\right\|=0 .
$$


To prove that the above condition is also a sufficient condition for the compactness of $T_{F G}-T_{F} T_{G}$, we need a certain distribution function inequality. The distribution function inequality involves the Lusin area integral and the Hardy-Littlewood maximal function. The idea to use distribution function inequalities in the theory of Toeplitz operators and Hankel operators first appeared in [2]. In this section we will get such a distribution function inequality.

For $w$ a point of $\partial D$, we let $\Gamma_{w}$ denote the angle with vertex $w$ and opening $\pi / 2$ which is bisected by the radius to $w$. The set of points z in $\Gamma_{w}$ satisfying $|z-w|<\epsilon$ will be denoted by $\Gamma_{w, \epsilon}$. For $\mathrm{h}$ in $L^{1}(\partial D)$, we define the Lusin area integral of $h$ to be

$$
A_{\epsilon}(h)(w)=\left[\int_{\Gamma_{w, \epsilon}}|\operatorname{grad} h(z)|^{2} d A(z)\right]^{1 / 2}
$$

where $\mathrm{h}(\mathrm{z})$ means the harmonic extension of $\mathrm{h}$ on $\mathrm{D}$ and $d A(z)$ denotes the Lebesgue measure on the unit disk $D$. The Hardy-Littlewood maximal function of the function $\mathrm{h}$ will be denoted $M h$, and for $r>1$, we let $\Lambda_{r} h=$ $\left[M|h|^{r}\right]^{1 / r}$. For $z \in D$, we let $I_{z}$ denote the closed subarc of $\partial D$ with center $\frac{z}{|z|}$ and measure $\delta(z)=1-|z|$. The Lebesgue measure of the subset $\mathrm{E}$ of $\partial D$ will be denoted by $|E|$.

Let $f_{i}$ and $g_{i}(i=1, \ldots, n)$ be in $L^{\infty}(\partial D)$ and $u$ and $v$ be in $H^{2}(\partial D)$. We define a generalized area integral to be

$$
B_{\epsilon}(u, v)(w)=\int_{\Gamma_{w, \epsilon}}\left|\sum_{i=1}^{n} \operatorname{grad}\left(H_{f_{i}} u\right) \overline{\operatorname{grad}\left(H_{g_{i}} v\right)}\right| d A(z) .
$$

For $l>2$ and $z \in D$, define

$$
\begin{array}{r}
\Xi_{l}(z)=\inf \left\{\sum_{i=1}^{n}\left(\left\|x_{i} \circ \phi_{z}-P\left(x_{i} \circ \phi_{z}\right)\right\|_{l}+\left\|y_{i} \circ \phi_{z}-P\left(g_{i} \circ \phi_{z}\right)\right\|_{l}\right):\right. \\
\left.A \in\left(M_{n \times n}\right)_{1}, \quad R \in P_{n}\right\},
\end{array}
$$

where

$$
\begin{aligned}
& x=\left(x_{1}, \cdots, x_{n}\right)^{T}=(R-A) f \text { and } y=\left(y_{1}, \ldots, y_{n}\right)^{T}=A^{*} g, \\
& \Gamma_{l}(z)=\sum_{i=1}^{n}\left(\left\|f_{i} \circ \phi_{z}-P\left(f_{i} \circ \phi_{z}\right)\right\|_{l}+\left\|g_{i} \circ \phi_{z}-P\left(g_{i} \circ \phi_{z}\right)\right\|_{l}\right) .
\end{aligned}
$$

We have the following distribution function inequality. 
Theorem 9 Let $f_{i}$ and $g_{i}$ be in $L^{\infty}, i=1, \cdots, n$, and $u$ and $v$ in the Hardy space $H^{2}$. Let $z$ be a point in $D$ such that $|z|>1 / 2$. Then for any $l>2$, for $a>0$ sufficiently large and $\delta(z)=1-|z|$

$$
\begin{aligned}
& \left|\left\{w \in I_{z}: B_{2 \delta(z)}(u, v)(w)<a \Xi_{l}(z) \Gamma_{l}(z) \inf _{w \in I_{z}} \Lambda_{r}(u)(w) \inf _{w \in I_{z}} \Lambda_{r}(v)(w)\right\}\right| \\
& \geq C_{a}\left|I_{z}\right|
\end{aligned}
$$

where $C_{a}$ depends only on $l$ and $a, \lim _{a \rightarrow \infty} C_{a}=1$, and $1 / l+1 / r=2 / p$ for some $1<p<2$ and $1<r<2$.

Proof. Let $f_{i}$ and $g_{i}$ be in $L^{\infty}$ and $u$ and $v$ in $H^{2}$. By our definition

$$
B_{\epsilon}(u, v)(w)=\int_{\Gamma_{w, \epsilon}}\left|\sum_{i=1}^{n} \operatorname{grad}\left(H_{f_{i}} u\right) \overline{\operatorname{grad}\left(H_{g_{i}} v\right)}\right| d A(w) .
$$

For a fixed $z \in D$, let $\chi_{2 I_{z}}$ denotes the characteristic function of the subset $2 I_{z}$ of $\partial D$ and write $H_{f_{i}} u=u_{i 1}+u_{i 2}, H_{g_{i}} v=v_{i 1}+v_{i 2}$, where

$$
\begin{aligned}
& u_{i 1}=(I-P)\left[\left(f_{i}-P\left(f \circ \phi_{z}\right)\right) \circ \phi_{z} \chi_{2 I_{z}} u\right], \\
& u_{i 2}=(I-P)\left[\left(f_{i}-P\left(f_{i} \circ \phi_{z}\right)\right) \circ \phi_{z}\left(1-\chi_{2 I_{z}}\right) u\right], \\
& v_{i 1}=(I-P)\left[\left(g_{i}-P\left(g_{i} \circ \phi_{z}\right)\right) \circ \phi_{z} \chi_{2 I_{z}} v\right], \\
& v_{i 2}=(I-P)\left[\left(g_{i}-P\left(g_{i} \circ \phi_{z}\right)\right) \circ \phi_{z}\left(1-\chi_{2 I_{z}}\right) v\right] .
\end{aligned}
$$

Thus we have

$$
B_{\epsilon}(u, v)(w) \leq \sum_{i=1}^{n}\left(A_{\epsilon}\left(u_{i 1}\right)+A_{\epsilon}\left(u_{i 2}\right)\right)\left(A_{\epsilon}\left(v_{i 1}\right)+A_{\epsilon}\left(v_{i 2}\right)\right) .
$$

From now on we use $\epsilon$ to denote $2 \delta(z)$. Let $i(1 \leq i \leq n)$ be fixed. We first recall the following estimates from [15] for the terms $A_{\epsilon}\left(u_{i 1}\right)$ and $A_{\epsilon}\left(u_{i 2}\right)$ (similarly $A_{\epsilon}\left(v_{i 1}\right)$ and $A_{\epsilon}\left(v_{i 2}\right)$ ).

For $l>2$, there are a positive constant $C$ and $r \in(1,2)$ such that

$$
\left[\int_{I_{z}} A_{\epsilon}\left(u_{i 1}\right)^{p} d \sigma(w)\right]^{1 / p} \leq C\left|I_{z}\right|^{1 / p}\left\|f_{i} \circ \phi_{z}-P\left(f_{i} \circ \phi_{z}\right)\right\|_{l} \inf _{w \in I_{z}} \Lambda_{r} u(w)
$$

for some $p>1$ so that $1 / l+1 / r=1 / p$.

For $l>2$, on $I_{z}$,

$$
A_{\epsilon}\left(u_{i 2}\right) \leq C\left\|f_{i} \circ \phi_{z}-P\left(f_{i} \circ \phi_{z}\right)\right\|_{l} \inf _{w \in I_{z}} \Lambda_{l^{\prime}} u(w),
$$

for some $C>0$ and $1 / l+1 / l^{\prime}=1$. 
For completeness we give a proof here. We first write $u_{i j}=(I-P) u_{j}$ $(j=1,2)$ where

$$
u_{1}=\left(f_{i-}-f_{i-}(z)\right) \chi_{2 I_{z}} u, \quad u_{2}=\left(f_{i-}-f_{i-}(z)\right)\left(1-\chi_{2 I_{z}} u\right)
$$

and $f_{i-}=(I-P) f_{i}$.

We first prove (7). Note that for $l>2$, we can always find $l^{\prime}>2$ and $p>1$ so that $l=l^{\prime} p$ and $r=p \frac{l^{\prime}}{l^{\prime}-1}<2$. By the theorem of Marcinkiewicz and Zygmund, the truncated Lusin area integral $A_{\epsilon} f(w)$ is $L^{p}$-bounded for $1<p<\infty$. So for $l>2$, we have

$$
\begin{aligned}
& \int_{I_{z}}\left[A_{\epsilon}(I-P)\left(u_{1}\right)(w)\right]^{p} d \sigma(w) \\
& \leq C \int_{\partial D}\left|u_{1}\right|^{p} d \sigma(w) \\
& =C \int_{2 I_{z}}\left|f_{i-}-f_{i-}(z)\right|^{p}|u(w)|^{p} d \sigma(w) \\
& \leq\left|2 I_{z}\right|\left[\frac{1}{\left|2 I_{z}\right|} \int_{2 I_{z}}\left|f_{i-}(w)-f_{i-}(z)\right|^{p l^{\prime}} d \sigma(w)\right]^{1 / l^{\prime}}\left[\frac{1}{\left|2 I_{z}\right|} \int_{2 I_{z}}|u|^{r} d \sigma(w)\right]^{p / r} .
\end{aligned}
$$

Let $P(z, w)$ denote the Poisson kernel for the point $z$. Since

$$
\left[\frac{1}{\left|2 I_{z}\right|} \int_{2 I_{z}}|u|^{r} d \sigma(w)\right]^{1 / r} \leq \Lambda_{r} u(w)
$$

for each $w \in 2 I_{z}$, and an elementary estimate shows that for $w \in 2 I_{z}$, $P(z, w)>\frac{C}{\left|2 I_{z}\right|}$, it follows that

$$
\begin{aligned}
& {\left[\int_{I_{z}} A_{\epsilon}\left((I-P)\left(u_{1}\right)(w)\right)^{p} d \sigma(w)\right]^{1 / p}} \\
& \leq C\left|I_{z}\right|^{1 / p}\left[\left|f_{i-}-f_{i-}(z)\right|^{l}(z)\right]^{1 / l} \inf _{w \in I_{z}} \Lambda_{r} u(w) .
\end{aligned}
$$

Thus (7) follows from the following identity.

$$
\left[\left|f_{i-}-f_{i-}(z)\right|^{l}(z)\right]^{1 / l}=\left\|f_{i} \circ \phi_{z}-P\left(f_{i} \circ \phi_{z}\right)\right\|_{l} .
$$

Now we prove (8). For $u_{2}$, we shall use a pointwise estimate of the norm of the gradient of $(I-P) u_{2}$. It is easy to see that

$$
(I-P)\left(u_{2}\right)(w)=\frac{1}{2 \pi} \int \frac{\bar{w} \xi u_{2}(\xi)}{1-\bar{w} \xi} d \sigma(\xi) .
$$

Thus

$$
\left|\operatorname{grad}(I-P) u_{2}(w)\right| \leq C \int \frac{\left|u_{2}(\xi)\right|}{|1-\bar{w} \xi|^{2}} d \sigma(\xi)
$$




$$
\leq C \int_{\partial D / 2 I_{z}} \frac{\left|\left[f_{i-}(\xi)-f_{i-}(z)\right] u(\xi)\right|}{|1-\bar{w} \xi|^{2}} d \sigma(\xi) .
$$

On the other hand, there is a constant $C>0$ so that

$$
\left|\frac{1-\bar{z} \xi}{1-\bar{w} \xi}\right| \geq C
$$

for all $\xi$ in $\partial D / 2 I_{z}$ and $w$ in $I_{z}$. Thus we obtain

$$
\left|\operatorname{grad}(I-P) u_{2}(w)\right| \leq C \int_{\partial D / 2 I_{z}} \frac{\left|\left[f_{i-}(\xi)-f_{i-}(z)\right] u(\xi)\right|}{|1-\bar{z} \xi|^{2}} d \sigma(\xi) .
$$

Applying the Hölder inequality yields

$$
\left|\operatorname{grad}(I-P) u_{2}(w)\right| \leq \frac{C}{1-|z|^{2}}\left[\left|f_{i-}-f_{i-}(z)\right|^{l}(z)\right]^{1 / l}\left[\left(|u|^{l^{\prime}}\right)(z)\right]^{1 / l^{\prime}} .
$$

Because the nontangential maximal function is bounded by a constant times the Hardy-Littlewood maximal function, and because $\mathrm{z}$ belongs to $\Gamma_{w, \epsilon}$, the last factor on the right is no larger than $\mathrm{C}_{l^{\prime}} u(w)$, and again the desired inequality is established by noting (9).

Now we can estimate the products $A_{\epsilon}\left(u_{i j}\right) A_{\epsilon}\left(v_{i j}\right), j=1,2$ by using (7) and (8). By Hölder inequality, we have

$$
\begin{aligned}
& {\left[\int_{I_{z}}\left[A_{\epsilon}\left(u_{i j}\right) A_{\epsilon}\left(v_{i j}\right)\right]^{p / 2} d \sigma(w)\right]} \\
& \leq\left[\int_{I_{z}}\left[A_{\epsilon}\left(u_{i j}\right)\right]^{p} d \sigma(w)\right]^{1 / 2}\left[\int_{I_{z}}\left[A_{\epsilon}\left(v_{i j}\right)\right]^{p} d \sigma(w)\right]^{1 / 2} .
\end{aligned}
$$

By using estimate (7) for integral of $A_{\epsilon}\left(u_{i 1}\right)$ or $A_{\epsilon}\left(v_{i 1}\right)$ and estimate (8) for integral of $A_{\epsilon}\left(u_{i 2}\right)$ or $A_{\epsilon}\left(v_{i 2}\right)$, we get that for $l>2$, there is $r$ with $1<r<2$ such that

$$
\begin{aligned}
& {\left[\int_{I_{z}}\left[A_{\epsilon}\left(u_{i j}\right) A_{\epsilon}\left(v_{i j}\right)\right]^{p / 2} d \sigma(w)\right]^{2 / p}} \\
& \leq C\left|I_{z}\right|^{2 / p}\left\|f_{i} \circ \phi_{z}-P\left(f_{i} \circ \phi_{z}\right)\right\|_{l}\left\|g_{i} \circ \phi_{z}-P\left(g_{i} \circ \phi_{z}\right)\right\|_{l} \\
& \quad \cdot \inf _{w \in I_{z}} \Lambda_{r} u(w) \inf _{w \in I_{z}} \Lambda_{r} v(w)
\end{aligned}
$$

for some $p>1$ so that $1 / l+1 / r=2 / p$, and a constant C depends on only $l$.

Now we are going to finish the proof by summing up the estimates as above. From (6) we have

$$
\left[\int_{I_{z}} B(u, v)^{p / 2} d \sigma(w)\right]^{2 / p} \leq C\left(\int_{I_{z}} \sum_{i=1}^{n} \sum_{j=1}^{2}\left[A_{\epsilon}\left(u_{i j}\right)(w) A_{\epsilon}\left(v_{i j}\right)(w)\right]^{p / 2}\right)^{2 / p} .
$$


By estimate (10), we have

$$
\begin{aligned}
& \int_{I_{z}} B(u, v)^{p / 2} d \sigma(w) \\
& \leq C\left|I_{z}\right|\left(\sum_{i=1}^{n}\left\|f_{i} \circ \phi_{z}-P\left(f_{i} \circ \phi_{z}\right)\right\|_{l}^{p / 2}\left\|g_{i} \circ \phi_{z}-P\left(g_{i} \circ \phi_{z}\right)\right\|_{l}^{p / 2}\right) \\
& \quad \cdot\left(\inf _{w \in I_{z}} \Lambda_{s} u(w) \inf _{w \in I_{z}} \Lambda_{s} v(w)\right)^{p / 2} \cdot
\end{aligned}
$$

Next for any $A \in\left(M_{n \times n}\right)_{1}$ and any permutation matrix $R \in P_{n}$, let

$$
x=\left(x_{1}, \cdots, x_{n}\right)^{T}=(R-A) f \text { and } y=\left(y_{1}, \cdots, y_{n}\right)^{T}=A^{*} g,
$$

where $f=\left(f_{1}, \cdots, f_{n}\right)^{T}$ and $g=\left(g_{1}, \cdots, g_{n}\right)^{T}$. We apply the above inequality (11) to the vector-valued functions $x$ and $y$. We note that the $B_{\epsilon}(u, v)$ corresponding to $f$ and $g$ is the same as the $B_{\epsilon}(u, v)$ corresponding to $x$ and $y$; more precisely,

$$
\begin{aligned}
& B_{\epsilon}(u, v)(w) \\
& =\int_{\Gamma_{w, \epsilon}}\left|\sum_{i=1}^{n} \operatorname{grad}\left(H_{f_{i}} u\right) \overline{\operatorname{grad}\left(H_{g_{i}} v\right)}\right| d A(w) \\
& =\int_{\Gamma_{w, \epsilon}}\left|\sum_{i=1}^{n}\left(\operatorname{grad}\left(H_{x_{i}} u\right) \overline{\operatorname{grad}\left(H_{g_{i}} v\right)}+\operatorname{grad}\left(H_{f_{i}} u\right) \overline{\operatorname{grad}\left(H_{y_{i}} v\right)}\right)\right| d A(w) .
\end{aligned}
$$

By formula (11), we have

$$
\begin{aligned}
& \int_{I_{z}} B(u, v)^{p / 2} d \sigma(w) \\
& \leq C\left|I_{z}\right|\left(\inf _{w \in I_{z}} \Lambda_{s} u(w) \inf _{w \in I_{z}} \Lambda_{s} v(w)\right)^{p / 2} \\
& \quad \cdot\left(\sum _ { i = 1 } ^ { n } \left(\left\|x_{i} \circ \phi_{z}-P\left(x_{i} \circ \phi_{z}\right)\right\|_{l}^{p / 2}\left\|g_{i} \circ \phi_{z}-P\left(g_{i} \circ \phi_{z}\right)\right\|_{l}^{p / 2}\right.\right. \\
& \left.\left.\quad+\left\|f_{i} \circ \phi_{z}-P\left(f_{i} \circ \phi_{z}\right)\right\|_{l}^{p / 2}\left\|y_{i} \circ \phi_{z}-P\left(y_{i} \circ \phi_{z}\right)\right\|_{l}^{p / 2}\right)\right) .
\end{aligned}
$$

Therefore

$$
\left[\int_{I_{z}} B_{\epsilon}(u, v)^{p / 2} d \sigma(w)\right]^{2 / p} \leq C\left|I_{z}\right|^{2 / p} \Xi_{l}(z) \Gamma_{l}(z) \inf _{w \in I_{z}} \Lambda_{s} u(w) \inf _{w \in I_{z}} \Lambda_{s} v(w) .
$$


Next for a fixed $\mathrm{z}$ in $\mathrm{D}$ and $a>0$, let $E(a)$ be the set of points in $I_{z}$ where

$$
B_{\epsilon}(u, v) \leq a \Xi_{l}(z) \Gamma_{l}(z) \inf _{w \in I_{z}} \Lambda_{s} h(w) \inf _{w \in I_{z}} \Lambda_{s} v(w) .
$$

Then

$$
\begin{aligned}
& \left|I_{z} / E(a)\right|^{2 / p} a \Xi_{l}(z) \Gamma_{l}(z) \inf _{w \in I_{z}} \Lambda_{s} h(w) \inf _{w \in I_{z}} \Lambda_{s} v(w) \\
& \leq\left[\int_{I_{z}} B_{\epsilon}(u, v) d \sigma(w)\right]^{2 / p} \\
& \leq C\left|I_{z}\right|^{2 / p} \Xi_{l}(z) \Gamma_{l}(z) \inf _{w \in I_{z}} \Lambda_{s} h(w) \inf _{w \in I_{z}} \Lambda_{s} v(w) .
\end{aligned}
$$

So

$$
\left|I_{z} / E(a)\right| \leq C a^{-p / 2}\left|I_{z}\right|
$$

Therefore for a sufficient large $a>0$, we have

$$
|E(a)| \geq\left(1-C a^{-p / 2}\right)\left|I_{z}\right|
$$

Let $C_{a}=1-C a^{-p / 2}$. This completes the proof of the theorem.

\section{Compact finite sum of products.}

Before proceeding to our main results in this section, we need to introduce some notations involving the maximal ideal space of an algebra. Let $\mathcal{M}$ be the maximal ideal space of $H^{\infty}$, which is defined to be the set of multiplicative linear maps from $H^{\infty}$ onto the field of complex numbers. Each multiplicative linear functional $\phi \in \mathcal{M}$ has norm 1 (as an element of the dual of $H^{\infty}$ ). If we think of $\mathcal{M}$ has a subset of the dual space $H^{\infty}$ with weak-star topology then $\mathcal{M}$ becomes a compact Hausdorff space. For $z \in D$ the evaluation functional $f \rightarrow f(z)$ is a multiplicative functional. So we can think of $D$ as a subset of $\mathcal{M}$. The Carleson corona theorem tells us that $D$ is dense in $\mathcal{M}$.

By using the Gelfand transform, we can think of $H^{\infty}$ as a subset of $C(\mathcal{M})$, the continuous, complex-valued functions on the maximal ideal space of $H^{\infty}$. Explicitly, for $f \in H^{\infty}$, we extend f from $D$ to $\mathcal{M}$ by defining

$$
f(\tau)=\tau(f)
$$

for every $\tau \in \mathcal{M}$. Note that this definition is consistent with our earlier identification of $D$ with a subset of $\mathcal{M}$.

By the Hahn-Banach theorem each $\tau \in \mathcal{M}$ extends to a linear functional $\tau^{\prime}$ on $L^{\infty}$. In fact, there is a unique representing measure $d \mu$ supported 
on $M\left(L^{\infty}\right)$, the maximal ideal space of $L^{\infty}$, such that for each $g \in L^{\infty}$, $\tau^{\prime}(g)=\int_{\operatorname{supp}\left(\tau^{\prime}\right)} g d \mu$. A subset of $M\left(L^{\infty}\right)$ will be called a support set, denoted by $\operatorname{supp} \tau$, if it is the (closed) support set of the representing measure for the extension of a functional $\tau$ in $M\left(H^{\infty}+C\right)$.

For $f \in L^{\infty}$, we let $H^{\infty}[f]$ denote the closed subalgebra of $L^{\infty}$ generated by $H^{\infty}$ and the function $f$. If $f=\left(f_{1}, \ldots, f_{n}\right)^{T}$, we still use $H^{\infty}[f]$ to denote the closed subalgebra of $L^{\infty}$ generated by $H^{\infty}$ and functions $f_{1}, \ldots, f_{n}$. Recall that

$$
\begin{array}{r}
\Xi_{2}(z)=\inf \left\{\sum_{i=1}^{n}\left(\left\|x_{i} \circ \phi_{z}-P\left(x_{i} \circ \phi_{z}\right)\right\|_{2}+\left\|y_{i} \circ \phi_{z}-P\left(g_{i} \circ \phi_{z}\right)\right\|_{2}\right):\right. \\
\left.A \in\left(M_{n \times n}\right)_{1} R \in P_{n}\right\},
\end{array}
$$

where

$$
x=\left(x_{1}, \cdots, x_{n}\right)^{T}=(R-A) f \text { and } y=\left(y_{1}, \ldots, y_{n}\right)^{T}=A^{*} g .
$$

Theorem 10 Let $f=\left(f_{1}, \ldots, f_{n}\right)^{T}$ and $g=\left(g_{1}, \ldots, g_{n}\right)^{T}$ for $f_{i}$ and $g_{i}$ in $L^{\infty}$. The following are equivalent.

(1) $H_{f_{1}}^{*} H_{g_{1}}+\cdots+H_{f_{n}}^{*} H_{g_{n}}$ is compact.

(2) $\lim _{z \rightarrow \partial D}\left\|\sum_{i=1}^{n}\left(H_{f_{i}} k_{z}\right) \otimes\left(H_{g_{i}} k_{z}\right)\right\|=0$.

(3) $\lim _{z \rightarrow \partial D} \Xi_{2}(z)=0$.

(4) For each $m \in M\left(H^{\infty}+C\right)$, there exist a matrix $A \in\left(M_{n \times n}\right)_{1}$ and a permutation matrix $R \in P_{n}$ such that $(R-A) f\left|\operatorname{supp} m \in H^{\infty}\right| \operatorname{supp} m$ and $A^{*} g\left|\operatorname{supp} m \in H^{\infty}\right| \operatorname{supp} m$.

(5) The following relation holds.

$$
\bigcap_{\left\{A \in\left(M_{n \times n}\right)_{1}, R \in P_{n}\right\}} H^{\infty}\left[(R-A) f, A^{*} g\right] \subset H^{\infty}+C .
$$

Proof. Without loss of generality we may assume that $\left\|f_{i}\right\|_{\infty}<1 / 2$ and $\left\|g_{i}\right\|_{\infty}<1 / 2$ for all $i=1, \ldots, n$.

$(1) \Longrightarrow(2)$. Assume that $\sum_{i=1}^{n} H_{f_{i}}^{*} H_{g_{i}}$ is compact. By Lemma $2[15]$ we obtain

$$
\lim _{|z| \rightarrow 1}\left\|\sum_{i=1}^{n} H_{f_{i}}^{*} H_{g_{i}}-T_{\phi_{z}}^{*}\left(\sum_{i=1}^{n} H_{f_{i}}^{*} H_{g_{i}}\right) T_{\phi_{z}}\right\|=0 .
$$

But by the proof of Lemma 1,

$$
V^{-1}\left(\sum_{i=1}^{n} H_{f_{i}}^{*} H_{g_{i}}-T_{\phi_{z}}^{*}\left(\sum_{i=1}^{n} H_{f_{i}}^{*} H_{g_{i}}\right) T_{\phi_{z}}\right) V=\sum_{i=1}^{n} H_{f_{i}} k_{z} \otimes H_{g_{i}} k_{z},
$$


where recall that $V$ is the antiunitary operator on $L^{2}(\partial \mathcal{D})$ defined by $V h\left(e^{i \theta}\right)$ $=e^{-i \theta} \overline{h\left(e^{i \theta}\right)}$. Thus

$$
\lim _{|z| \rightarrow 1}\left\|\sum_{i=1}^{n} H_{f_{i}} k_{z} \otimes H_{g} k_{z}\right\|=0 .
$$

That is (2) holds.

$(2) \Longrightarrow(3)$. Assume now that (2) holds. Suppose that (3) does not hold. That is, there are $\delta>0$ and a net $\{z\} \subset D$ accumulating a point in $\partial D$ such that

$$
\Xi_{2}(z) \geq \delta
$$

We will get a contradiction. We may assume that the net $\{z\}$ converges to some nontrivial point $m \in M\left(H^{\infty}+C\right)$.

Let $H_{m}^{\infty}$ denote the algebra $\left.H^{\infty}\right|_{\operatorname{supp} m}$ on $\operatorname{supp} m$, and $L_{m}^{\infty}$ denote the algebra $\left.L^{\infty}\right|_{\text {supp } m}$. Then $L_{m}^{\infty} / H_{m}^{\infty}$ is a vector space. For a function $\psi$ in $L^{\infty}$, let $[\psi]_{m}$ denote the element in $L_{m}^{\infty} / H_{m}^{\infty}$ which contains $\psi$. For $f=$ $\left(f_{1}, \ldots, f_{n}\right)^{T}$, let $[f]_{m}=\left(\left[f_{1}\right]_{m}, \ldots,\left[f_{n}\right]_{m}\right)^{T}$ and $f \in H_{m}^{\infty}$ means that $f_{i} \in$ $H_{m}^{\infty}$ for all $i=1, \ldots, n$. Let $g=\left(g_{1}, \ldots, g_{n}\right)^{T}$. For convenience we also introduce the following notations.

$$
\begin{gathered}
\left\|H_{f} k_{z}\right\|_{2}:=\sum_{i=1}^{n}\left\|H_{f_{i}} k_{z}\right\|_{2}, \\
H_{f} k_{z} \otimes H_{g} k_{z}:=\sum_{i=1}^{n} H_{f_{i}} k_{z} \otimes H_{g_{i}} k_{z} .
\end{gathered}
$$

Suppose that the dimension of the space spanned by $\left[f_{1}\right]_{m}, \ldots,\left[f_{n}\right]_{m}$ is $N \leq n$. We may assume that $\left\{\left[f_{1}\right]_{m}, \ldots,\left[f_{N}\right]_{m}\right\}$ is a basis such that $\left(\left[f_{1}\right]_{m}, \ldots,\left[f_{n}\right]_{m}\right)^{T}=B\left(\left[f_{1}\right]_{m}, \ldots,\left[f_{N}\right]_{m}\right)^{T}$ with $B=\left(b_{i j}\right)$ and $\left|b_{i j}\right| \leq 1$, see the proof of Proposition 4 for details. Let $A$ be the matrix $(B, 0)_{n \times n}$. Then $f-A f$ is in $H_{m}^{\infty}$ on the support set $\operatorname{supp} m$. By Lemma 3 [15],

$$
\lim _{z \rightarrow m}\left\|H_{f-A f} k_{z}\right\|_{2}=0 .
$$

On the other hand,

$$
\begin{aligned}
H_{f} k_{z} \otimes H_{g} k_{z} & =H_{f-A f} k_{z} \otimes H_{g} k_{z}+H_{f} k_{z} \otimes H_{A^{*} g} k_{z} \\
& =H_{f-A f} k_{z} \otimes H_{g} k_{z}+H_{f_{(N)}} k_{z} \otimes H_{B^{*} g} k_{z}
\end{aligned}
$$

where $f_{(N)}=\left(f_{1}, \ldots, f_{N}\right)$. As $z$ goes to $m$, the first term in the right hand side of the above equation goes to zero. Hence

$$
\lim _{z \rightarrow m}\left\|H_{f_{(N)}} k_{z} \otimes H_{B^{*} g} k_{z}\right\|=0 .
$$


We are going to show that

$$
\lim _{z \rightarrow m}\left\|H_{B^{*} g} k_{z}\right\|_{2}=0 .
$$

Suppose that this is not true. We may assume that

$$
\lim _{z \rightarrow m}\left\|H_{g_{1}} k_{z}\right\|_{2}>0 .
$$

Let $a_{i}(z)=\left\langle H_{g_{1}} k_{z}, H_{g_{i}} k_{z}\right\rangle$. Note that $\left|a_{i}(z)\right| \leq \sqrt{\left\|g_{1}\right\|_{\infty}} \sqrt{\left\|g_{i}\right\|_{\infty}} \leq 1$. We may assume that $a_{i}(z)$ converges to $a_{i}$ as $z$ goes to $m$. By our assumption $a_{1} \neq 0$. But

$$
\lim _{z \rightarrow m}\left\|\left(H_{f_{(N)}} k_{z} \otimes H_{B^{*} g} k_{z}\right) H_{g_{1}} k_{z}\right\|_{2}=0
$$

implies that

$$
\lim _{z \rightarrow m}\left\|H_{\sum_{i=1}^{N} a_{i} f_{i}} k_{z}\right\|_{2}=0 .
$$

By Lemma 3 [15], $\sum_{i=1}^{N} a_{i} f_{i}$ is in $H^{\infty}$ on suppm. This contradicts the fact that $\left\{\left[f_{1}\right]_{m}, \ldots,\left[f_{N}\right]_{m}\right\}$ is a basis. Therefore

$$
\lim _{z \rightarrow m}\left\|H_{B^{*} g} k_{z}\right\|_{2}=0
$$

Hence

$$
\lim _{z \rightarrow m}\left\|H_{A^{*} g} k_{z}\right\|_{2}=0
$$

But

$$
\left\|H_{(I-A) f} k_{z}\right\|+\left\|H_{A^{*} g} k_{z}\right\| \geq \Xi_{2}(z) .
$$

Hence

$$
\lim _{z \rightarrow m}\left(\left\|H_{(I-A) f} k_{z}\right\|+\left\|H_{A^{*} g} k_{z}\right\|\right) \geq \delta .
$$

This is a contradiction.

$(3) \Longrightarrow(4)$. We are going to show that for each $m \in M\left(H^{\infty}+C\right)$, there exist matrices $A_{m} \in\left(M_{n \times n}\right)_{1}$ and $R_{m} \in P_{n}$ such that

$$
\left[\left(R_{m}-A_{m}\right) f\right]_{m}=0,\left[A_{m}^{*} g\right]_{m}=0 .
$$

Let $z$ be a net in $D$ converging to $m$. By condition (3), there are matrices $A_{z} \in\left(M_{n \times n}\right)_{1}$ and $R_{z} \in P_{n}$ such that

$$
\lim _{z \rightarrow m}\left(\left\|H_{\left(R_{z}-A_{z}\right) f} k_{z}\right\|_{2}+\left\|H_{A_{z}^{*} g} k_{z}\right\|_{2}\right)=0 .
$$


Since $\left(M_{n \times n}\right)_{1}$ is compact and the permutation group $P_{n}$ is also compact, we may assume that $A_{z}$ converges to $A_{m}$ and $R_{z}$ converges to $R_{m}$. Hence

$$
\lim _{z \rightarrow m}\left(\left\|H_{\left(R_{m}-A_{m}\right) f} k_{z}\right\|_{2}+\left\|H_{A_{m}^{*} g} k_{z}\right\|_{2}\right)=0 .
$$

By Lemma 3 [15], we have

$$
\left[\left(R_{m}-A_{m}\right) f\right]_{m}=0, \quad\left[A_{m}^{*} g\right]_{m}=0 .
$$

$(4) \Longrightarrow(5)$. By the Chang-Marshall theorem [8], we need only to show that

$$
M\left(H^{\infty}+C\right) \subset M\left(\cap_{\left\{A \in\left(M_{n \times n}\right)_{1}, R \in P_{n}\right\}} H^{\infty}\left[(R-A) f, A^{*} g\right]\right) .
$$

Condition (4) states exactly that

$$
M\left(H^{\infty}+C\right) \subset \cup_{\left\{A \in\left(M_{n \times n}\right)_{1}, R \in P_{n}\right\}} M\left(H^{\infty}\left[(R-A) f, A^{*} g\right]\right) .
$$

By the Sarason theorem [11],

$$
\begin{aligned}
& M\left(\cap_{\left\{A \in\left(M_{n \times n}\right)_{1}, R \in P_{n}\right\}} H^{\infty}\left[(R-A) f, A^{*} g\right]\right) \\
& =\text { Closure of } \cup_{\left\{A \in\left(M_{n \times n}\right)_{1}, R \in P_{n}\right\}} M\left(H^{\infty}\left[(R-A) f, A^{*} g\right]\right) .
\end{aligned}
$$

Hence

$$
M\left(H^{\infty}+C\right) \subset M\left(\cap_{\left\{A \in\left(M_{n \times n}\right)_{1}, R \in P_{n}\right\}} H^{\infty}\left[(R-A) f, A^{*} g\right]\right) .
$$

$(5) \Longrightarrow(3)$. Suppose that (3) does not hold. There are $\delta>0$ and a net $z$ in $D$ converging to some $m \in M\left(H^{\infty}+C\right)$ such that

$$
\Xi_{2}(z) \geq \delta
$$

By condition (5) and Sarason's Theorem [11] as in (14), there are a net $m_{\alpha} \in M\left(H^{\infty}\right)$ and matrices $A_{\alpha}, R_{\alpha}$ such that $m_{\alpha}$ converges to $m$ and

$$
\left[\left(R_{\alpha}-A_{\alpha}\right) f\right]_{m_{\alpha}}=0,\left[A_{\alpha}^{*} g\right]_{m_{\alpha}}=0 .
$$

We may assume that $A_{\alpha}$ converges to some $A_{m}$ and $R_{\alpha}$ converges to some $R_{m}$. We claim that

$$
\left[\left(R_{m}-A_{m}\right) f\right]_{m}=0,\left[A_{m}^{*} g\right]_{m}=0 .
$$

As in Lemma 3 [15], let $u_{i}(i=1, \ldots, n)$ be the unimodular functions such that $u_{m}=\left(u_{1}, \ldots, u_{n}\right)^{T}$ in $\left(R_{m}-A_{m}\right) f+H_{n}^{\infty}$ and $v_{i}(i=1, \cdots, n)$ be 
the unimodular functions such that $v_{m}=\left(v_{1}, \ldots, v_{n}\right)^{T}$ in $A_{m}^{*} g+H_{n}^{\infty}$. Then by Lemma 3 [15]

$$
\begin{aligned}
& \sum_{i=1}^{n}\left[\left(1-\left|u_{i}(z)\right|^{2}\right)+\left(1-\left|v_{i}(z)\right|^{2}\right)\right] \\
& \leq C\left[\left\|A_{\alpha}-A_{m}\right\|_{\infty}+\left\|R_{\alpha}-R_{m}\right\|_{\infty}\right]+C\left[\left\|H_{\left(R_{\alpha}-A_{\alpha}\right) f} k_{z}\right\|_{2}+\left\|H_{A_{\alpha}^{*} g} k_{z}\right\|_{2}\right]
\end{aligned}
$$

for all $z \in D$. Hence

$$
\sum_{i=1}^{n}\left[\left(1-\left|u_{i}\left(m_{\alpha}\right)\right|^{2}\right)+\left(1-\left|v_{i}\left(m_{\alpha}\right)\right|^{2}\right)\right] \leq C\left[\left\|A_{\alpha}-A_{m}\right\|_{\infty}+\left\|R_{\alpha}-R_{m}\right\|_{\infty}\right] .
$$

Since these functions $u_{i}$ and $v_{i}$ are continuous on $M\left(H^{\infty}\right)$, we have

$$
\sum_{i=1}^{n}\left[\left(1-\left|u_{i}(m)\right|^{2}\right)+\left(1-\left|v_{i}(m)\right|^{2}\right)\right]=0 .
$$

Therefore

$$
\left[\left(R_{m}-A_{m}\right) f\right]_{m}=0,\left[A_{m}^{*} g\right]_{m}=0 .
$$

This proves our claim. But again by Lemma 3 [15], this implies that

$$
\lim _{z \rightarrow m}\left(\left\|H_{\left(R_{m}-A_{m}\right) f} k_{z}\right\|_{2}+\left\|H_{A_{m}^{*} g} k_{z}\right\|_{2}\right)=0 .
$$

This contradicts to the assumption that

$$
\left\|H_{\left(R_{m}-A_{m}\right) f} k_{z}\right\|_{2}+\left\|H_{A_{m}^{*} g} k_{z}\right\|_{2} \geq \Xi_{2}(z)>\delta .
$$

$(3) \Longrightarrow(1)$. Now we assume that

$$
\lim _{z \rightarrow \partial D} \Xi_{2}(z)=0
$$

We use the distribution inequality obtained in Section 5 to show that $\sum_{i=1}^{n} H_{f_{i}}^{*} H_{g_{i}}$ is compact. Since the quantity $\Xi_{r}(z)$ for some $r>2$ appears in the distribution inequality, we first need to show that in fact for some $r$ such that $3>r>2$,

$$
\lim _{z \rightarrow \partial D} \Xi_{r}(z)=0
$$

Recall that

$$
\Xi_{l}(z)=\inf \left\{\sum_{i=1}^{n}\left(\left\|x_{i} \circ \phi_{z}-P\left(x_{i} \circ \phi_{z}\right)\right\|_{l}+\left\|y_{i} \circ \phi_{z}-P\left(g_{i} \circ \phi_{z}\right)\right\|_{l}\right):\right.
$$




$$
\left.A \in\left(M_{n \times n}\right)_{1} R \in P_{n}\right\}
$$

where

$$
x=\left(x_{1}, \cdots, x_{n}\right)^{T}=(R-A) f \text { and } y=\left(y_{1}, \cdots, y_{n}\right)^{T}=A^{*} g .
$$

First note that since $A \in\left(M_{n \times n}\right)_{1}, R \in P_{n}$ and by our assumption $\left\|f_{i}\right\|_{\infty}<$ $1 / 2$ and $\left\|g_{i}\right\|_{\infty}<1 / 2$, we have $\left\|x_{i}\right\|_{\infty} \leq(1+n) / 2$ and $\left\|y_{i}\right\|_{\infty} \leq n / 2$. Thus

$$
\begin{aligned}
& \sum_{i=1}^{n}\left(\left\|x_{i} \circ \phi_{z}-P\left(x_{i} \circ \phi_{z}\right)\right\|_{r}+\left\|y_{i} \circ \phi_{z}-P\left(y_{i} \circ \phi_{z}\right)\right\|_{r}\right) \\
& \leq C_{r}\left(\sum_{i=1}^{n}\left(\left\|x_{i} \circ \phi_{z}-P\left(x_{i} \circ \phi_{z}\right)\right\|_{2}+\left\|y_{i} \circ \phi_{z}-P\left(y_{i} \circ \phi_{z}\right)\right\|_{2}\right)^{2 / r}\right),
\end{aligned}
$$

for some constant $C_{r}$ dependent only on $r$ and $n$. Therefore

$$
\Xi_{r}(z) \leq C_{r}\left(\Xi_{2}(z)\right)^{2 / r}
$$

Since $\lim _{z \rightarrow \partial D} \Xi_{2}(z)=0$, we obtain

$$
\lim _{z \rightarrow \partial D} \Xi_{r}(z)=0 .
$$

This completes the proof of (15).

Now let $u$ and $v$ be two functions in $H^{2}$. Since $H_{f_{i}} u$ is orthogonal to $H^{2}$, we see that $\left(H_{f_{i}} u\right)(0)=0$. Thus by the Littlewood-Paley formula [8], we have

$$
\begin{aligned}
& \left\langle u,\left(\sum_{i=1}^{n} H_{f_{i}}^{*} H_{g_{i}}\right) v\right\rangle=\sum_{i=1}^{n}\left\langle H_{f_{i}} u, H_{g_{i}} v\right\rangle \\
& =\frac{1}{\pi} \int_{\mathcal{D}}\left(\sum_{i=1}^{n} \operatorname{grad}\left(H_{f_{i}} u\right) \overline{\operatorname{grad}\left(H_{g_{i}} v\right)}\right) \log \frac{1}{|z|} d A(z)=I_{R}+I I_{R},
\end{aligned}
$$

where for $1 / 2<R<1$,

$$
I_{R}=\int_{|z|>R}\left(\sum_{i=1}^{n} \operatorname{grad}\left(H_{f_{i}} u\right) \overline{\operatorname{grad}\left(H_{g_{i}} v\right)}\right) \log \frac{1}{|z|} d A(z)
$$

and

$$
I I_{R}=\int_{|z| \leq R}\left(\sum_{i=1}^{n} \operatorname{grad}\left(H_{f_{i}} u\right) \overline{\operatorname{grad}\left(H_{g_{i}}\right)}\right) \log \frac{1}{|z|} d A(z)
$$


One easily checks that there is a compact operator $K_{R}$ such that

$$
I I_{R}=\left\langle u, K_{R} v\right\rangle .
$$

Thus, if we show that $I_{R} \rightarrow 0$ as $R \rightarrow 1$, then $\left\|T_{f} T_{g}-T_{g} T_{f}-K_{R}\right\| \rightarrow 0$, and we are done. The rest of the proof will be devoted to showing that $I_{R} \rightarrow 0$ as $R \rightarrow 1$.

Choose $z \in \mathcal{D}$ and fix a constant $a \geq 1$ for which the Distribution Inequality holds; that is

$$
\left|t \in I_{z}:\left\{B_{\epsilon}(u, v)(t) \leq a \Xi_{r}(z) \Gamma_{r}(z) \Lambda_{s} u(t) \Lambda_{s} v(t)\right\}\right| \geq C_{a}\left|I_{z}\right| .
$$

For $t \in \partial \mathcal{D}$, let

$$
\rho(t)=\max \left\{\epsilon: B_{\epsilon}(u, v)(t) \leq a \Xi_{r}(z) \Gamma_{r}(z) \Lambda_{s} u(t) \Lambda_{s} v(t)\right\} .
$$

Let $\chi_{t}$ denote the characteristic function of $\Gamma_{t, \rho(t)}$. Then

$$
\begin{aligned}
\int_{\partial \mathcal{D}} B_{\rho(t)}(u, v)(t) d t & \leq a \Xi_{r}(z) \Gamma_{r}(z) \int_{\partial \mathcal{D}} \Lambda_{s} u(t) \Lambda_{s} v(t) d t \\
& \leq a \Xi_{r}(z) \Gamma_{r}(z)\left\|\Lambda_{s} u\right\|_{2}\left\|\Lambda_{s} v\right\|_{2} .
\end{aligned}
$$

Since $\frac{2}{s}>1$, so by $[8]$

$$
\left\|\Lambda_{s} u\right\|_{2}=\left\|M\left(|u|^{s}\right)^{1 / s}\right\|_{2}=\left[\left\|M\left(|u|^{s}\right)\right\|_{2 / s}\right]^{1 / s} \leq A_{s}\left(\left\||u|^{s}\right\|_{2 / s}\right)^{1 / s} .
$$

So

$$
\left\|\Lambda_{s} u\right\|_{2} \leq A_{s}\|u\|_{2}
$$

Similarly,

$$
\left\|\Lambda_{s} v\right\|_{2} \leq A_{s}^{\prime}\|v\|_{2}
$$

Thus

$$
\int_{\partial \mathcal{D}} B_{\rho(t)}(u, v)(t) d t \leq a^{*} \Xi_{r}(z) \Gamma_{r}(z)\|u\|_{2}\|v\|_{2} .
$$

On the other hand,

$$
\int_{\partial \mathcal{D}} B_{\epsilon}(u, v)(t) d t=\int_{\partial \mathcal{D}} \int_{\Gamma_{t, \rho(t)}}\left|\sum_{i=1}^{n} \operatorname{grad}\left(H_{f_{i}} u\right) \overline{\operatorname{grad}\left(H_{g_{i}} v\right)}\right| d A(z) d t .
$$

So

$$
\int_{\partial \mathcal{D}} B_{\epsilon}(u, v)(t) \geq \int_{\partial \mathcal{D}} \int_{|z|>R} \chi_{t}(z)\left|\sum_{i=1}^{n} \operatorname{grad}\left(H_{f_{i}} u\right) \overline{\operatorname{grad}\left(H_{g_{i}} v\right)}\right| d A(z) d t .
$$


Now the Distribution Function Inequality tells us that $\rho(t) \geq\left(1-|z|^{2}\right)$ on a subset $E_{z}$ of $I_{z}$ satisfying

$$
\left|E_{z}\right| \geq C_{a}\left|I_{z}\right|
$$

Now, for $t \in E_{z}$, we have $t \in I_{z}$. Thus if we write $z=r e^{i \theta}$ and note that $\rho(t) \geq \frac{3}{2}(1-|z|)$ we have

$$
\left.\left|r e^{i \theta}-e^{i t}\right| \leq\left|r e^{i \theta}-e^{i \theta}\right|+\left|e^{i \theta}-e^{i t}\right| \leq(1-|z|)+\frac{(1-|z|)}{2}\right) \leq \rho(t) .
$$

Therefore, for $t \in E_{z}$, we have that $z \in \Gamma_{t, \rho(t)}$ and that $\chi_{t}(z)=1$ on $E_{z}$. So,

$$
\begin{aligned}
& \int_{\partial \mathcal{D}} \int_{|z|>R} \chi_{t}(z)\left|\sum_{i=1}^{n} \operatorname{grad}\left(H_{f_{i}} u\right) \overline{\operatorname{grad}\left(H_{g_{i}} v\right)}\right| d A(z) d t \\
& \geq \int_{|z|>R}\left[\int_{\partial \mathcal{D}} \chi_{t}(z) d t\right]\left|\sum_{i=1}^{n} \operatorname{grad}\left(H_{f_{i}} u\right) \overline{\operatorname{grad}\left(H_{g_{i}} v\right)}\right| d A(z) .
\end{aligned}
$$

Since $\chi_{t}(z)=1$ on $E_{z}$, we have

$$
\int_{\partial \mathcal{D}} B_{\epsilon}(u, v)(t) d t \geq \int_{|z|>R}\left|E_{z}\right|\left|\sum_{i=1}^{n} \operatorname{grad}\left(H_{f_{i}} u\right) \overline{\operatorname{grad}\left(H_{g_{i}} v\right)}\right| d A(z) .
$$

But, $\left|E_{z}\right| \geq C_{a}\left(1-|z|^{2}\right)$, so

$$
\int_{\partial \mathcal{D}} B_{\epsilon}(u, v)(t) \geq C_{a} \int_{|z|>R}\left|\sum_{i=1}^{n} \operatorname{grad}\left(H_{f_{i}} u\right) \overline{\operatorname{grad}\left(H_{g_{i}} v\right)}\right|\left(1-|z|^{2}\right) d A(z) .
$$

Since

$$
I_{R}=\int_{|z|>R}\left|\sum_{i=1}^{n} \operatorname{grad}\left(H_{f_{i}} u\right) \overline{\operatorname{grad}\left(H_{g_{i}} v\right)}\right| \log \frac{1}{|z|} d A(z)
$$

we have,

$$
\int_{\partial \mathcal{D}} B_{\epsilon}(u, v)(t) \geq C_{a}\left|I_{R}\right|
$$

Combining this together with (16), we see that

$$
\left|I_{R}\right| \leq C \Xi_{r}(z) \Gamma_{r}(z)\|u\|_{2}\|v\|_{2} .
$$

But by (15),

$$
\lim _{z \rightarrow \partial D} \Xi_{r}(z)=0
$$

and $\Gamma_{r}(z)$ is bounded. Hence we have $\lim _{R \rightarrow 1}\left|I_{R}\right|=0$. This finishes the proof. 


\section{Compact semi-commutator or commutator.}

In this section by combining the results in Sections 2 and 6, we will show several necessary and sufficient conditions for the semi-commutator or the commutator of the block Toeplitz operators with matrix symbols to be compact. We also give a characterization of essentially normal block Toeplitz operators.

Theorem 11. Let $F$ and $G$ be in $L_{n \times n}^{\infty}$. Let $F^{*}=\left(f_{1}, \ldots, f_{n}\right)$ and $G=\left(g_{1}, \ldots, g_{n}\right)$. The following are equivalent.

(1) $T_{F G}-T_{F} T_{G}\left(=H_{F^{*}}^{*} H_{G}\right)$ is compact.

$$
\lim _{|z| \rightarrow 1}\left\|\left[\left|\left(F_{+}\right)^{*}-\left(F_{+}\right)^{*}(z)\right|^{2}(z)\right]^{1 / 2}\left[\left|G_{-}-G_{-}(z)\right|^{2}(z)\right]^{1 / 2}\right\|=0 .
$$

Proof. (1) $\Longleftrightarrow(2)$. Let $F=\left(f_{i j}\right)_{n \times n}$ and $G=\left(g_{i j}\right)_{n \times n}$. Note that $T_{F G}-$ $T_{F} T_{G}$ is compact if and only if each entry of

$$
\left(\sum_{k} H_{f_{i k}}^{*} H_{g_{k j}}\right)_{i j}
$$

is compact. By Theorem 10, this is equivalent to

$$
\cup_{i, j} \cap_{\left\{A \in\left(M_{n \times n}\right)_{1}, R \in P_{n}\right\}} H^{\infty}\left[(R-A) f_{i}, A^{*} g_{j}\right] \subset H^{\infty}+C .
$$

$(1) \Longleftrightarrow(3)$. (1) $\Rightarrow(3)$ is proved in Theorem 3 . We are going to prove that $(3) \Rightarrow(1)$. By Lemma 2 ,

$$
\begin{aligned}
& \operatorname{tr}\left(\left|\left(F_{+}\right)^{*}-\left(F_{+}\right)^{*}(z)\right|^{2}(z)\right)\left(\left|G_{-}-G_{-}(z)\right|^{2}(z)\right) \\
& =\operatorname{trace}\left[H_{F^{*}}^{*} H_{G}-T_{\Phi_{z}}^{*} H_{F^{*}}^{*} H_{G} T_{\Phi_{z}}\right]^{*}\left[H_{F^{*}}^{*} H_{G}-T_{\Phi_{z}}^{*} H_{F^{*}}^{*} H_{G} T_{\Phi_{z}}\right] .
\end{aligned}
$$

Thus by Lemma 1, (3) implies that

$$
\lim _{|z| \rightarrow 1}\left\|\sum_{k} H_{\overline{f_{i k}}} k_{z} \otimes H_{g_{k j}} k_{z}\right\|=0
$$

for all $i, j$. By Theorem 10, we have that $\sum_{k} H_{f_{i k}}^{*} H_{g_{k j}}$ are compact for all $i, j$. Hence $H_{F^{*}}^{*} H_{G}$ is compact. Therefore $T_{F G}-T_{F} T_{G}$ is compact. The proof is complete.

Next we characterize when the commutator $T_{F} T_{G}-T_{G} T_{F}$ is compact. To do this, recall that

$$
T_{F} T_{G}-T_{G} T_{F}=T_{F} T_{G}-T_{F G}+T_{G F}-T_{G} T_{F}+T_{(F G-G F)}
$$




$$
=-\left(H_{F^{*}}^{*} H_{G}-H_{G^{*}}^{*} H_{F}\right)+T_{(F G-G F)} .
$$

Therefore by the Douglas theorem [7], $T_{F} T_{G}-T_{G} T_{F}$ is compact if and only if $F G=G F$ and $H_{F^{*}}^{*} H_{G}-H_{G^{*}}^{*} H_{F}$ is compact. But if we let

$$
B=\left[\begin{array}{cc}
F & -G \\
0 & 0
\end{array}\right], \quad C=\left[\begin{array}{cc}
G & 0 \\
F & 0
\end{array}\right],
$$

then

$$
H_{B^{*}}^{*} H_{C}=\left[\begin{array}{cc}
H_{F^{*}}^{*} H_{G}-H_{G^{*}}^{*} H_{F} & 0 \\
0 & 0
\end{array}\right] .
$$

Therefore, $T_{F} T_{G}-T_{G} T_{F}$ is compact if and only if $F G=G F$ and $H_{B^{*}}^{*} H_{C}$ is compact. Note that $\left|\left(B_{+}\right)^{*}-\left(B_{+}\right)^{*}(z)\right|^{2}(z)$ and $\left|C_{-}-C_{-}(z)\right|^{2}(z)$ can be computed as in (4) and (5). The following result now follows immediately from Theorem 11.

Theorem 12. Let $F$ and $G$ be in $L_{n \times n}^{\infty}$. Let

$$
\left[\begin{array}{c}
F^{*} \\
-G^{*}
\end{array}\right]=\left(f_{1}, \ldots, f_{n}\right), \quad\left[\begin{array}{c}
G \\
F
\end{array}\right]=\left(g_{1}, \cdots, g_{n}\right) .
$$

The following are equivalent.

(1) $T_{F} T_{G}-T_{G} T_{F}$ is compact.

(2) $F G=G F$ and $\cup_{i, j} \cap_{\left\{A \in\left(M_{n \times n}\right)_{1}, R \in P_{n}\right\}} H^{\infty}\left[(R-A) f_{i}, A^{*} g_{j}\right] \subset H^{\infty}+C$.

(3) $F G=G F$ and

$$
\begin{gathered}
\lim _{|z| \rightarrow 1} \|\left[\begin{array}{cc}
\left|\left(F_{+}\right)^{*}-\left(F_{+}\right)^{*}(z)\right|^{2}(z) & -\left(\left(\left(F_{+}\right)^{*}-\left(F_{+}\right)^{*}(z)\right)\right. \\
-\left(\left(\left(G_{+}\right)^{*}-\left(G_{+}\right)^{*}(z)\right)\right. & \left.\mid\left(G_{+}-G_{+}(z)\right)\right)(z) \\
\left.\cdot\left(F_{+}-F_{+}(z)\right)\right)(z) & \left|\left(G_{+}\right)^{*}-\left(G_{+}\right)^{*}(z)\right|^{2}(z)
\end{array}\right]^{1 / 2} \\
\cdot\left[\begin{array}{cc}
\left|G_{-}-G_{-}(z)\right|^{2}(z) & \left(( G _ { - } - G _ { - } ( z ) ) \left(\left(F_{-}\right)^{*}\right.\right. \\
\left(( F _ { - } - F _ { - } ( z ) ) \left(\left(G_{-}\right)^{*}\right.\right. & \left.\left.-\left(F_{-}\right)^{*}(z)\right)\right)(z) \\
\left.\left.-\left(G_{-}\right)^{*}(z)\right)\right)(z) & \left|F_{-}-F_{-}(z)\right|^{2}(z)
\end{array}\right]^{1 / 2} \|=0 .
\end{gathered}
$$

An operator $A$ is said to be essentially normal if $A^{*} A-A A^{*}$ is compact. By taking $G=F^{*}$, we immediately get the following characterization of essentially normal block Toeplitz operators.

Corollary 13 Let $F$ be in $L_{n \times n}^{\infty}$. Let

$$
\left[\begin{array}{c}
F^{*} \\
-F
\end{array}\right]=\left(f_{1}, \cdots, f_{n}\right), \quad\left[\begin{array}{c}
F^{*} \\
F
\end{array}\right]=\left(g_{1}, \cdots, g_{n}\right) .
$$


The following are equivalent.

(1) $T_{F}$ is essentially normal.

(2) $F F^{*}=F^{*} F$ and $\cup_{i, j} \cap_{\left\{A \in\left(M_{n \times n}\right)_{1}, R \in P_{n}\right\}} H^{\infty}\left[(R-A) f_{i}, A^{*} g_{j}\right] \subset H^{\infty}+C$.

(3) $\quad F F^{*}=F^{*} F$ and

$$
\begin{aligned}
\lim _{|z| \rightarrow 1} \|\left[\begin{array}{ll}
\left|\left(F_{+}\right)^{*}-\left(F_{+}\right)^{*}(z)\right|^{2}(z) & -\left(\left(F_{-}-F_{-}(z)\right)\right. \\
-\left(\left(\left(F_{+}\right)^{*}-\left(F_{+}\right)^{*}(z)\right)\right. & \left.\cdot\left(F_{+}-F_{+}(z)\right)\right)(z) \\
\left.\cdot\left(\left(F_{-}\right)^{*}-\left(F_{-}\right)^{*}(z)\right)\right)(z) & \left|F_{-}-F_{-}(z)\right|^{2}(z)
\end{array}\right]^{1 / 2} \\
\qquad\left[\begin{array}{ll}
\left|\left(F_{+}\right)^{*}-\left(F_{+}\right)^{*}(z)\right|^{2}(z) & \left(\left(F_{-}-F_{-}(z)\right)\right. \\
\left(\left(\left(F_{+}\right)^{*}-\left(F_{+}\right)^{*}(z)\right)\right. & \left.\left(F_{+}(z)\right)\right)(z) \\
\left.\cdot\left(\left(F_{-}\right)^{*}-\left(F_{-}\right)^{*}(z)\right)\right)(z) & \left|F_{-}-F_{-}(z)\right|^{2}(z)
\end{array}\right]^{1 / 2} \|=0 .
\end{aligned}
$$

For the scalar symbols there were several other sufficient conditions for the product of two Toeplitz operators to be a compact perturbation of a Toeplitz operator. To state those conditions we need some notations. The fiber $M_{\lambda}$ of $M\left(L^{\infty}\right)$ above the point $\lambda$ is the set $\left\{\tau \in M\left(L^{\infty}\right): z(\tau)=\lambda\right\}$. We recall that a subset of $M\left(L^{\infty}\right)$ is called an antisymmetric set if any real-valued function in $H^{\infty}+C$ is constant on the set.

One of the following conditions implies the compactness of the semicommutator $T_{\bar{\phi}} T_{\psi}-T_{\bar{\phi} \psi}$ of Toeplitz operators with scalar symbols $\phi$ and $\psi$.

(1) Either $\phi$ or $\psi$ is in $C(\partial D)[5]$.

(2) $\phi$ and $\psi$ are piecewise continuous and have no common discontinuities [10]

(3) Either $\bar{\phi}$ or $\psi$ is in $H^{\infty}$ on each fiber $M_{z}$ for $z$ on the circle [13].

(4) Either $\bar{\phi}$ is in $H^{\infty}$ or $\psi$ is in $H^{\infty}$ on each set of maximal antisymmetry of $H^{\infty}+C$ [1]. It was shown in [2] that $H^{\infty}[\bar{\phi}] \cap H^{\infty}[\psi] \subset H^{\infty}+C$ is equivalent to

(5) Either $\phi$ or $\psi$ is in $H^{\infty}$ on each support set.

Next we will show some sufficient conditions for the compactness of the semi-commutator $T_{F} T_{G}-T_{F G}$ of Toeplitz operators with matrix symbols $F$ and $G$. Those conditions are analogous to the above conditions of the scalar case. Some of them are well known ([7], [9]).

Corollary 14. Let $F$ and $G$ be in $L_{n \times n}^{\infty}$. Then one of the following conditions is a sufficient condition for $T_{F} T_{G}-T_{F G}$ to be compact:

(1) Either $F^{*}$ or $G$ is in $C_{n \times n}(\partial D)$. 
(2) $F^{*}$ and $G$ are piecewise continuous and have no common discontinuities.

(3) Either $F^{*}$ or $G$ is in $H_{n \times n}^{\infty}$ on each fiber $M_{z}$ for $z$ on the circle.

(4) Either $F^{*}$ or $G$ is in $H_{n \times n}^{\infty}$ on each maximal antisymmetric set of $H^{\infty}+C$.

(5) Either $F^{*}$ or $G$ is in $H_{n \times n}^{\infty}$ on each support set.

(6) $H_{n \times n}^{\infty}\left[F^{*}\right] \cap H_{n \times n}^{\infty}[G] \subset H_{n \times n}^{\infty}+C_{n \times n}(\partial D)$, where $H_{n \times n}^{\infty}[G]$ denotes the subalgebra of $L_{n \times n}^{\infty}$ generated by $H_{n \times n}^{\infty}$ and $G$.

Proof. Notice that Conditions (1) to (6) in the corollary are ordered by weakness. So it is sufficient to show that Condition (6) is stronger than Condition (2) in Theorem 11. Since $H_{n \times n}^{\infty}[G]$ denotes the subalgebra of $L_{n \times n}^{\infty}$ generated by $H_{n \times n}^{\infty}$ and $G$, we observe that

$$
H_{n \times n}^{\infty}[G]=\left(H^{\infty}\left[g_{11}, \ldots, g_{1 n}, \ldots, g_{n 1}, \ldots, g_{n n}\right]\right)_{n \times n} .
$$

Hence Condition (6) is equivalent to

$$
\begin{aligned}
H^{\infty}\left[\overline{f_{11}}, \ldots, \overline{f_{1 n}}, \ldots, \overline{f_{n 1}}, \ldots, \overline{f_{n n}}\right] \cap H^{\infty}\left[g_{11}, \ldots,\right. \\
\left.g_{1 n}, \ldots, g_{n 1}, \ldots, g_{n n}\right] \subset H^{\infty}+C .
\end{aligned}
$$

Let $F^{*}=\left(f_{1}, \ldots, f_{n}\right)$ and $G=\left(g_{1}, \ldots, g_{n}\right)$. It is easy to see that

$$
\cup_{i, j} \cap_{\left\{A \in\left(M_{n \times n}\right)_{1}, R \in P_{n}\right\}} H^{\infty}\left[(R-A) f_{i}, A^{*} g_{j}\right] \quad \subset \cup_{i, j} H^{\infty}\left[f_{i}\right] \cap H^{\infty}\left[g_{j}\right] .
$$

But for all $i, j$, we have

$$
\begin{gathered}
H^{\infty}\left[f_{i}\right] \cap H^{\infty}\left[g_{j}\right] \\
\subset H^{\infty}\left[\overline{f_{11}}, \ldots, \overline{f_{1 n}}, \ldots, \overline{f_{n 1}}, \ldots, \overline{f_{n n}}\right] \cap H^{\infty}\left[g_{11}, \ldots, g_{1 n}, \cdots, g_{n 1}, \ldots, g_{n n}\right] .
\end{gathered}
$$

Hence

$$
\cup_{i, j} \cap_{\left\{A \in\left(M_{n \times n}\right)_{1}, R \in P_{n}\right\}} H^{\infty}\left[(R-A) f_{i}, A^{*} g_{j}\right] \subset H^{\infty}+C .
$$

So by Theorem 11, $T_{F} T_{G}-T_{F G}$ is compact. This completes the proof.

Acknowledgment. We thank S. Treil for his useful suggestions.

\section{References}

[1] S. Axler, Doctoral dissertation, University of California, Berkeley, 1975. 
[2] S. Axler, S.-Y.A. Chang and D. Sarason, Product of Toeplitz operators, Integral Equations and Operator Theory, 1 (1978), 285-309.

[3] A. Böttcher and B. Silbermann, Analysis of Toeplitz operators, Springer-Verlag, 1990.

[4] A. Brown and P.R. Halmos, Algebraic properties of Toeplitz operators, J. Reine Angew. Math., 213 (1963), 89-102.

[5] L.A. Coburn, The $C^{*}$-algebra generated by an isometry I, Bull. Amer. Math. Soc., 137 (1967), 211-217.

[6] R.G. Douglas, Banach algebra techniques in the operator theory, Academic Press, New York and London, 1972.

[7] R. G. Douglas, Banach algebra techniques in the theory of Toeplitz operators, Regional Conference Series in Mathematics, American Mathematical Society 15, 1972.

[8] J. B. Garnett, Bounded Analytic Functions, Academic Press, New York, 1981.

[9] I. Gohberg, S. Goldberg and M.A. Kaashoek, Classes of Linear Operators, Vol. 2, Birkhauser Verlag Basel, 1993.

[10] I.C. Gohberg and N.J. Krupnik, The algebra generated by Toeplitz matrices, Functional Anal. Appl., 3 (1969), 119-127.

[11] P. Gorkin and D. Zheng, Essentially commuting Toeplitz operators, preprint.

[12] P.R. Halmos, A Hilbert space problem book, New York, Springer-Verlag, 1982.

[13] D. Sarason, On products of Toeplitz operators, Acta Scien. Math., 35 (1973), 7-12.

[14] A. Volberg, Two remarks concerning the theorem of S. Axler, S.-Y.A. Chang and D. Sarason, J. Operator Theory, 8 (1982), 209-218.

[15] D. Zheng, The distribution function inequality and products of Toeplitz operators and Hankel operators, J. Functional Analysis, 138 (1996), 477-501.

Received January 3, 1997. The first author was supported in part by NSF grant DMS90022140 during residence at MSRI. The second author was supported in part by the National Science Foundation.

California Polytechnic State University

SAn Luis OBISPo, CA 93407

E-mail address: cgu@oboe.calpoly.edu

VANDERBILT UNIVERSITY

NASHVILle, TN 37240

E-mail address: zheng@math.vanderbilt.edu 